\title{
GAUSSIAN FLUCTUATIONS FOR THE CLASSICAL XY MODEL
}

\author{
CHARLES M. NEWMAN, WEI WU
}

\begin{abstract}
We study the classical XY model in bounded domains of $\mathbb{Z}^{d}$ with Dirichlet boundary conditions. We prove that when the temperature goes to zero faster than a certain rate as the lattice spacing goes to zero, the fluctuation field converges to a standard Gaussian white noise. This and related results also apply to a large class of gradient field models.
\end{abstract}

\section{INTRODUCTION}

In this paper we study the classical XY model (also known as the classical rotator model) in dimension $d \geq 2$. Assign to each vertex $i \in \mathbb{Z}^{d}$ a spin $s(i) \in S^{1}$ with the corresponding angle $\theta(i) \in[-\pi, \pi)$. The XY model is defined formally as a Gibbs measure with Hamiltonian given by

$$
H=-\sum_{i \sim j} s(i) \cdot s(i)=-\sum_{i \sim j} \cos (\theta(i)-\theta(j))
$$

where the sum is over all nearest neighbor pairs of vertices. It is an example of the more general $O(n)$ model, where each spin takes a value in the sphere $S^{n-1}$.

A simple heuristic discussion of the low temperature behavior of the $\mathrm{XY}$ model is as follows. As the temperature goes to zero, the spins tend to align with each other so as to minimize the Hamiltonian. Since $\cos (\delta \theta) \approx 1-(\delta \theta)^{2} / 2$ for small $\delta \theta$, it is expected that at low temperature, the XY Gibbs measure on large scales behaves like a Gaussian Free Field (GFF). This idea originated in Dys56] (see also [MW66]) and was referred to as the Gaussian spin-wave approximation. By further making connections between rotational symmetry of the XY model and the recurrence/transience property of simple random walks, it was proved in [MW66] that for $d \leq 2$, there is no spontaneous magnetization at any strictly positive temperature. A related argument was applied in [FSS76] to show that for $d \geq 3$, with appropriate boundary conditions, the spin configuration has a preferred direction at low temperature - i.e., there is spontaneous magnetization, but has no spontaneous magnetization at high temperature (and thus there is a phase transition).

For $d=2$, the Gaussian spin-wave approximation is expected to be valid for all temperatures below the critical temperature corresponding to the so-called Kosterlitz-Thouless transition (see [KT73]). This suggests, for example, that the scaling limits, as the lattice spacing tends to zero, of the XY and GFF models on the lattice, should be closely related to each other. But this has not been proved for any fixed positive temperature. The best known results are only for the two point correlation functions: a polynomial upper bound for the spin-spin correlation function was proved in [MS77], and a different polynomial lower bound was established in [FS81]. A more modest question, which is to show that at some fixed low temperature, the spin-spin correlation has an asymptotic power law decay, still remains open. For $d \geq 3$, it was proved that at low temperature the spin configuration has long range order by using the infrared bound [FSS76] and also proved that the two point (transverse) correlation function has a power law decay [BFL ${ }^{+80}$ ]. Yet 
it is still an open problem to match the powers in the upper and lower bound for the longitudinal correlation function (the cosine-cosine correlation).

In this paper we make some modest progress about the Gaussian spin-wave approximation. We study the XY model in finite domains in $\varepsilon \mathbb{Z}^{d}$, for $d \geq 2$, with Dirichlet boundary conditions, where the inverse temperature $\beta$ depends on $\varepsilon$, and is required to grow at least proportional to $|\log \varepsilon|$ as $\varepsilon$ tends to zero (see Section 2 for precise conditions). With this assumption, the spin configuration has a preferred direction even for $d=2$, and our results focus on the fluctuation field. We prove that the rescaled (properly defined) gradient field of the XY model converges weakly to a Gaussian white noise. This suggests that the spin field should converge to some version of a compactified GFF (i.e., a GFF modulo $2 \pi$ ).

The significance of our requirement on $\beta(\varepsilon)$ growing fast enough as $\varepsilon \rightarrow 0$ is that when $\beta(\varepsilon)$ is proportional to $|\log \varepsilon|$, applying a result of [BF81] (see our Theorem 51) shows that on an event with high probability, there are no vortices (see Section 2 for the definition) in a domain of size $\varepsilon^{-1}$. This allows one to construct a coupling between the XY model and a corresponding convex gradient field model, such that their gradient variables agree on an event with high probability. It then relates the scaling limit of the XY model to the corresponding limit for a gradient field with a convex potential, where the situation is much better understood because of the Helffer-Sjöstrand formula ([HS94], see also our Section 4.1) that represents the covariance of the local functionals of the field in terms of a random walk in random conductances. We mention [NS97, GOS01 and Mil11 as an incomplete list of literature regarding central limit theorem type results for such convex gradient fields. We adapt the arguments in these papers to the current setting where the potential depends on the lattice spacing, in order to prove our fluctuation results. We further show that the limiting quadratic form has intensity one - i.e., the white noise scaling limit corresponds to the gradient of a standard GFF.

The rest of this paper is organized as follows. In Section 2 we set up the model and state the main results (Theorems 2 and 4). Section 3 starts with a contour estimate for the XY model (Theorem 5) from [BF81, and a corresponding result for the gradient field (Proposition 17); it then uses these estimates to construct a coupling between the two measures. The rest of the paper is then devoted to central limit theorem (CLT) type results for the corresponding gradient field model. We first prove a CLT type result in all of $\varepsilon \mathbb{Z}^{d}$ (in Section 44) and then in bounded domains (in Section 5). Combined with the coupling established in Section 3 we complete the proof of our main results. Finally, Section 6 discusses two open questions and related conjectures.

\section{Main Results}

Fix $d \geq 2$. Let $D \subset \mathbb{R}^{d}$ be a bounded domain with smooth boundary, $D^{\varepsilon}=D \cap \varepsilon \mathbb{Z}^{d}$. We also denote by $\left(D^{\varepsilon}\right)^{*}$ the set of all nearest neighbor directed edges $(x, y)$ with $x$ and $y$ in $D^{\varepsilon}$. We will have a further assumption on the $\left(D^{\varepsilon}\right)^{*}$ graph and hence on $D$ below. Given $x \in D^{\varepsilon}$, we associate an angular variable $\theta^{\varepsilon}(x) \in[-\pi, \pi)$, and a spin variable $s^{\varepsilon}(x)=\left(\cos \theta^{\varepsilon}(x), \sin \theta^{\varepsilon}(x)\right)$. Given $\beta>0$, the classical XY model on $D^{\varepsilon}$ is defined by the following Gibbs measure $\mu_{\beta}^{\varepsilon}$ on $[-\pi, \pi)^{D^{\varepsilon}}\left(\right.$ or $\left.\left(S^{1}\right)^{D^{\varepsilon}}\right)$ :

$$
d \mu_{\beta}^{\varepsilon}=Z_{\beta}^{-1} \exp \left[\beta \sum_{(i, j) \in\left(D^{\varepsilon}\right)^{*}} \cos \left(\theta^{\varepsilon}(i)-\theta^{\varepsilon}(j)\right)\right] \prod_{i \in D^{\varepsilon} \backslash \partial D^{\varepsilon}} d \theta^{\varepsilon}(i) \prod_{i \in \partial D^{\varepsilon}} \delta_{0}\left(d \theta^{\varepsilon}(i)\right),
$$


where $Z_{\beta}$ is the normalizing constant. For any directed edge $b=(i, j) \in\left(D^{\varepsilon}\right)^{*}$, we define the variable

$$
\eta^{\varepsilon}(b)=\left\{\begin{array}{cc}
\theta^{\varepsilon}(j)-\theta^{\varepsilon}(i)+2 \pi & \text { if } \theta^{\varepsilon}(j)-\theta^{\varepsilon}(i) \in(-2 \pi,-\pi) \\
\theta^{\varepsilon}(j)-\theta^{\varepsilon}(i) & \text { if } \theta^{\varepsilon}(j)-\theta^{\varepsilon}(i) \in[-\pi, \pi) \\
\theta^{\varepsilon}(j)-\theta^{\varepsilon}(i)-2 \pi & \text { if } \theta^{\varepsilon}(j)-\theta^{\varepsilon}(i) \in[\pi, 2 \pi)
\end{array} .\right.
$$

In other words, $\eta^{\varepsilon}(b)$ is the smallest increment (in absolute value) that deforms $\theta^{\varepsilon}(i)$ to $\theta^{\varepsilon}(j)(\bmod 2 \pi)$. For $l=1, \ldots, d$ and $x \in D^{\varepsilon}$, we will sometimes denote $\eta_{l}^{\varepsilon}(x)=$ $\eta^{\varepsilon}\left(x, x+\varepsilon e_{l}\right)$, where $e_{l}$ is the $l^{\text {th }}$ standard basis vector in $\mathbb{R}^{d}$ or $\mathbb{Z}^{d}$. The Hamiltonian of the XY model (more precisely, $\beta$ times the Hamiltonian) can also be written as $H(\eta)=$ $-\beta \sum_{b \in\left(D^{\varepsilon}\right)^{*}} \cos \left(\eta^{\varepsilon}(b)\right)$.

We see that $\left\{\eta^{\varepsilon}\right\}$ satisfies the following hardcore constraint:

$$
\eta^{\varepsilon}\left(b_{1}\right)+\eta^{\varepsilon}\left(b_{2}\right)+\eta^{\varepsilon}\left(b_{3}\right)+\eta^{\varepsilon}\left(b_{4}\right)=2 k_{P} \pi
$$

whenever $\left(b_{1}, b_{2}, b_{3}, b_{4}\right)$ encloses a plaquette $P$ in $D^{\varepsilon}$, where $k_{P}=0, \pm 1, \pm 2$. If $k_{P} \neq 0$, we call $P$ a vortex of the spin configuration $\left\{\theta^{\varepsilon}\right\}$. $\eta^{\varepsilon}$ differs from the usual (discrete) gradient of $\theta^{\varepsilon}$, which is just $\theta^{\varepsilon}(j)-\theta^{\varepsilon}(i)$ for $(i, j) \in\left(D^{\varepsilon}\right)^{*}$. When $\left\{\theta^{\varepsilon}\right\}$ is "vortex free", i.e., $\eta^{\varepsilon}\left(b_{1}\right)+\eta^{\varepsilon}\left(b_{2}\right)+\eta^{\varepsilon}\left(b_{3}\right)+\eta^{\varepsilon}\left(b_{4}\right)=0$ for every plaquette $P$, we want $\eta^{\varepsilon}$ to be gradient of some function $\Phi$, such that for any $x \in D^{\varepsilon}, \theta^{\varepsilon}(x)=\Phi(x) \bmod 2 \pi$.

For the vortex free condition to imply the existence of such a $\Phi$, one wishes to define $\Phi(x)=\sum_{b \in \mathcal{C}_{x^{*} \rightarrow x}} \eta^{\varepsilon}(b)$, where $\mathcal{C}_{x^{*} \rightarrow x}$ is any path in $\left(D^{\varepsilon}\right)^{*}$ connecting $x^{*}$ to $x$ and conclude that $\eta^{\varepsilon}=\nabla \Phi$. For this to work it is necessary that $\Phi$ is well defined, i.e., that the vortex condition implies that the formula for $\Phi$ does not depend on the choice of $\mathcal{C}_{x^{*} \rightarrow x}$. This will be so if $\left(D^{\varepsilon}\right)^{*}$ satisfies the following topological requirement.

Definition 1. We say that $\left(D^{\varepsilon}\right)^{*}$ is simply connected if for any site self-avoiding loop $b$, there is a finite sequence of loops $b_{1}=b, b_{2}, \ldots, b_{N}$ with $b_{j}$ and $b_{j-1}$ differing (algebraically) by a single plaquette for each $j$, such that $b_{N}$ is a trivial loop with no edge. We say that the domain $D$ in $\mathbb{R}^{d}$ is discretely simply connected if for each $\varepsilon>0$ such that $\left(D^{\varepsilon}\right)^{*}$ is nonempty, $\left(D^{\varepsilon}\right)^{*}$ is simply connected.

Henceforth we assume that $D$ is discretely simply connected. This, for example, is the case for $D$ a rectangular parallelipiped along the coordinate axis, $\left[a_{1}, b_{1}\right] \times \ldots \times\left[a_{d}, b_{d}\right]$ with $a_{j}<b_{j}$ for each $j$.

We study the zero temperature limit of the Gibbs measure (1), by letting $\beta=\beta(\varepsilon)$ depend on the lattice size $\varepsilon$ with $\beta(\varepsilon) \rightarrow \infty$ as $\varepsilon \rightarrow 0$. More precisely, we assume that,

$$
\beta(\varepsilon)+9 d \log \varepsilon \rightarrow \infty \text { as } \varepsilon \rightarrow 0 .
$$

It is straightforward to see that with this choice of $\beta(\varepsilon)$, the spins $\left\{\theta^{\varepsilon}(x)\right\}_{x \in D^{\varepsilon}}$ (and the associated gradient variables $\left.\eta^{\varepsilon}(b), b \in\left(D^{\varepsilon}\right)^{*}\right)$ concentrate around 0 as $\varepsilon$ tends to zero (for a quantitative bound, see Theorem 5 below). We focus on the fluctuation field of the $\left\{\eta^{\varepsilon}\right\}$ variables. To do this, for $b \in\left(D^{\varepsilon}\right)^{*}$, we define rescaled variables by setting $\tilde{\eta}^{\varepsilon}(b)=\sqrt{\beta(\varepsilon)} \eta^{\varepsilon}(b)$. Our main result is the following.

Theorem 2. Suppose that $\tilde{\eta}^{\varepsilon}=\sqrt{\beta(\varepsilon)} \eta^{\varepsilon}$ is the fluctuating field associated with the Gibbs measure (1), and suppose $\beta(\varepsilon)$ satisfies (4). Define the linear functional

$$
\left\langle\tilde{\eta}^{\varepsilon}, \varphi\right\rangle=\varepsilon^{(d / 2)-1} \sum_{b \in\left(D^{\varepsilon}\right)^{*}} \nabla \varphi(b) \tilde{\eta}^{\varepsilon}(b), \text { for } \varphi \in \mathcal{C}_{0}^{\infty}(D)
$$


where $\nabla \varphi(b)=\varphi(y)-\varphi(x)$ for $b=(x, y)$. Then for any $t \in \mathbb{R}$,

$$
\lim _{\varepsilon \rightarrow 0} \mathbb{E}\left[e^{i t\left\langle\tilde{\eta}^{\varepsilon}, \varphi\right\rangle}\right]=\exp \left[-\frac{t^{2}}{2}\langle\partial \varphi, \partial \varphi\rangle\right]
$$

where

$$
\langle\partial \varphi, \partial \varphi\rangle=\int_{D} \sum_{\alpha=1}^{d}\left(\frac{\partial \varphi}{\partial x_{\alpha}}\right)^{2} d x
$$

Remark 3. By a standard approximation argument (see, e.g. Mil11), one can strengthen the topology of convergence and replace the space of test functions in Theorem 2 by $H^{\kappa}(D)$, the Sobolev space of degree $\kappa$, for every $\kappa>4$.

The argument to prove Theorem 2 is fairly robust, and as we see in the next theorem, it applies to a large class of gradient field models. We say $\left\{\theta^{\varepsilon}(\cdot)\right\} \in \mathbb{R}^{D^{\varepsilon}}$ is a zero boundary gradient field on $D^{\varepsilon}$ with potential $V: \mathbb{R} \rightarrow \mathbb{R}$, if its distribution is the Gibbs measure

$$
d \nu_{\beta}=Z_{\beta}^{-1} \exp \left[-\beta \sum_{(i, j) \in\left(D^{\varepsilon}\right)^{*}} V\left(\theta^{\varepsilon}(i)-\theta^{\varepsilon}(j)\right)\right] \prod_{i \in D^{\varepsilon} \backslash \partial D^{\varepsilon}} d \theta^{\varepsilon}(i) \prod_{i \in \partial D^{\varepsilon}} \delta_{0}\left(d \theta^{\varepsilon}(i)\right),
$$

where $Z_{\beta}$ is the normalizing constant. For any directed edge $b=(i, j) \in\left(D^{\varepsilon}\right)^{*}$, define the associated gradient field $\eta^{\varepsilon}(b)=\theta^{\varepsilon}(j)-\theta^{\varepsilon}(i)$.

Theorem 4. Suppose that $\eta^{\varepsilon}$ is the gradient field associated with the Gibbs measure (7), such that $V$ is symmetric, $V^{\prime \prime}$ is Lipschitz, and $\inf _{x \in \mathbb{R}} V^{\prime \prime}(x)>0$. Also suppose $\beta=$ $\beta(\varepsilon)$ satisfies (4). For convenience, we assume that $\tilde{\theta}^{\varepsilon}$ (and $\tilde{\eta}^{\varepsilon}$ ) has been rescaled by a multiplicative constant so that $V^{\prime \prime}(0)=1$. Then the same conclusion as in Theorem 2 holds.

Theorem 4 can be proved by the same argument as Theorem 2, only replacing the Peierls type estimate (Theorem [5) by Proposition 17. Taking $V(x)=\frac{1}{2} x^{2}+\lambda x^{4}$, for $\lambda>0$, Theorem 4 applies to anharmonic crystal models (see, e.g., GK80]). Moreover, for gradient field models with periodic boundary conditions, one can prove a version of Theorem 4 for more general potentials $V(\cdot)$. Indeed, it suffices to have $V \geq 0$ satisfying the conditions in Theorem 4 in a neighborhood of 0 , and such that $\int_{-\infty}^{\infty} e^{-b V(x)} d x<\infty$ for any $b>0$. The proof under this more general assumption replaces Proposition 7 by a similar estimate using reflection positivity [Shl86] (see also [Bis09]).

Below, when there is no danger of confusion, we will omit some super and subscripts. For example, we will denote $\tilde{\eta}^{\varepsilon}$ as $\eta$, and $\mu_{\beta}^{\varepsilon}$ as $\mu$, etc.

\section{Coupling to CONVEX GRAdient Fields}

In this section, we discuss coupling the XY model to convex gradient fields. The coupling will be used at the end of Section 5 to prove Theorem 2.

3.1. Contour estimates. The following estimate for the angular variables in the classical $\mathrm{XY}$ model is due to Bricmont and Fontaine [BF81, the proof of which makes use of the Ginibre inequality. This is a continuous spin analogue of the Peierls estimate for discrete spin models.

Theorem 5 ([BF81]). There exists a constant $c<\infty$ such that, for any $\varepsilon>0$, any $a \in(0, \pi]$ and any collection $\mathcal{C}$ of edges $b \in\left(D^{\varepsilon}\right)^{*}$ such that if $(i, j) \in \mathcal{C}$ then $(j, i) \notin \mathcal{C}$,

$$
\mathbb{E}\left[\prod_{b \in \mathcal{C}} 1_{\left|\eta^{\varepsilon}(b)\right|>a}\right] \leq\left(c \exp \left(-\beta a^{2} / \pi^{2}\right)\right)^{|\mathcal{C}|} .
$$


Therefore if we fix any $\delta>0$, then as $\beta \rightarrow \infty$, it is very unlikely to find a large set of edges, such that the gradient variable $\left|\eta^{\varepsilon}(\cdot)\right|$ on each of these edges is larger than $\delta$. It is thus natural to believe that if we modify the cosine potential in (11) outside a $\delta$-neighborhood of 0 , the large scale behavior of the new gradient field will be the same as for (1), in the $\beta \rightarrow \infty$ limit.

Given $0<\delta<\pi / 2$, define a convex potential $V^{\delta}: \mathbb{R} \rightarrow \mathbb{R}$ by

$$
V^{\delta}(x)=\left\{\begin{array}{cc}
-\cos x & |x| \leq \delta \\
-\cos \delta+\sin \delta \cdot(x-\delta)+\frac{\cos \delta}{2}(x-\delta)^{2} & x>\delta \\
-\cos \delta-\sin \delta \cdot(x+\delta)+\frac{\cos \delta}{2}(x+\delta)^{2} & x<-\delta
\end{array} .\right.
$$

Notice that $\inf _{x \in \mathbb{R}}\left(V^{\delta}\right)^{\prime \prime}(x)=\cos \delta$. Now let $\mu_{\beta}^{\varepsilon, \delta}$ be the gradient Gibbs measure defined by

$d \mu_{\beta}^{\varepsilon, \delta}=\left(Z_{\beta}^{\delta}\right)^{-1} \exp \left[-\beta \sum_{(i, j) \in\left(D^{\varepsilon}\right)^{*}} V^{\delta}\left(\theta^{\varepsilon, \delta}(i)-\theta^{\varepsilon, \delta}(j)\right)\right] \prod_{i \in D^{\varepsilon} \backslash \partial D^{\varepsilon}} d \theta^{\varepsilon, \delta}(i) \prod_{i \in \partial D^{\varepsilon}} \delta_{0}\left(d \theta^{\varepsilon, \delta}(i)\right)$.

Let $\mathbb{E}^{\delta}$ denote the expectation with respect to $\mu_{\beta}^{\varepsilon, \delta}$.

We now state a contour estimate for the measure $\mu_{\beta}^{\varepsilon, \delta}$. The proof uses the following version of the Brascamp-Lieb inequality that applies to gradient Gibbs measures with convex potential.

Lemma 6 (Brascamp-Lieb inequality BL76]). Let $D$ be a finite graph. Let $\mu$ be a gradient Gibbs measure on $D$ defined as follows with $V_{e}^{\prime \prime}$ continuous on $\mathbb{R}$ for each $e \in D^{*}$ :

$$
d \mu=Z^{-1} \exp \left[-\sum_{e=(i, j) \in D^{*}} V_{e}(\theta(i)-\theta(j))\right] \prod_{i \in D \backslash \partial D} d \theta(i) \prod_{i \in \partial D} \delta_{0}(d \theta(i)) .
$$

For $e=(i, j) \in D^{*}$, let $\eta(e)=\theta(i)-\theta(j)$. Let $c_{-}=\inf _{e \in D^{*}} \inf _{x \in \mathbb{R}} V_{e}^{\prime \prime}(x)$ and assume $c_{-}>0$. Then the following two inequalities are valid.

1. For all $e \in D^{*}, \operatorname{Var}_{\mu}(\eta(e)) \leq c_{-}^{-1}$.

2. For any $t>0$ and any $\varphi \in \mathbb{R}^{D}$,

$$
\mathbb{E}\left[e^{t\langle\eta, \varphi\rangle}\right] \leq \exp \left(\frac{t^{2}}{2}\left\langle\nabla \varphi, c_{-}^{-1} \nabla \varphi\right\rangle\right)
$$

where $\langle\eta, \varphi\rangle=\sum_{e \in D^{*}} \eta(e) \nabla \varphi(e)$.

Proposition 7. For any $\varepsilon>0$, any $0<a<\delta \in(0, \pi / 3]$, any collection $\mathcal{C}$ of edges $b=(i, j) \in\left(D^{\varepsilon}\right)^{*}$ such that if $(i, j) \in \mathcal{C}$ then $(j, i) \notin \mathcal{C}$, and any $\beta \geq 0$,

$$
\mathbb{E}^{\delta}\left[\prod_{(i, j) \in \mathcal{C}} 1_{\left|\theta^{\varepsilon, \delta}(j)-\theta^{\varepsilon, \delta}(i)\right|>a}\right] \leq\left(\exp \left(1-\beta a^{2} / \pi^{2}\right)\right)^{|\mathcal{C}|}
$$


Proof. To simplify notation, we write $\theta^{\varepsilon, \delta}$ as $\theta, \eta^{\varepsilon, \delta}$ as $\eta, \mu_{\beta}^{\varepsilon, \delta}$ as $\mu$, and $Z^{\delta}$ as $Z$. Note that

$$
\begin{aligned}
& \mathbb{E}^{\delta}\left[\prod_{(i, j) \in \mathcal{C}} 1_{|\theta(j)-\theta(i)|>a}\right] \\
= & \mathbb{E}^{\delta}\left[\prod_{(i, j) \in \mathcal{C}} 1_{|\theta(j)-\theta(i)|>a} \exp \left[\frac{\beta}{2} V^{\delta}(\theta(j)-\theta(i))\right] \exp \left[-\frac{\beta}{2} V^{\delta}(\theta(j)-\theta(i))\right]\right] \\
\leq & \exp \left[\frac{\beta}{2}\left(1-\frac{2 a^{2}}{\pi^{2}}\right)|\mathcal{C}|\right] \mathbb{E}^{\delta} \prod_{(i, j) \in \mathcal{C}} \exp \left[\frac{\beta}{2} V^{\delta}(\theta(j)-\theta(i))\right],
\end{aligned}
$$

because $\cos x \leq 1-\frac{2 a^{2}}{\pi^{2}}$ for $0 \leq a \leq|x| \leq \pi / 3$.

To apply the Brascamp-Lieb inequality (Lemma 6) and obtain an upper bound for

$$
\mathbb{E}^{\delta} \prod_{(i, j) \in \mathcal{C}} \exp \left[\frac{\beta}{2} V^{\delta}(\theta(j)-\theta(i))\right],
$$

we first note that since $V^{\delta}(x) \leq x^{2} / 2-1$ (as can easily be checked by comparing second derivatives) and hence

$$
\begin{aligned}
\mathbb{E}^{\delta} \prod_{(i, j) \in \mathcal{C}} \exp \left[\frac{\beta}{2} V^{\delta}(\theta(j)-\theta(i))\right] & \leq \mathbb{E}^{\delta} \prod_{b \in \mathcal{C}} \exp \left[\frac{\beta}{2}\left(\frac{\eta(b)^{2}}{2}-1\right)\right] \\
& =\exp \left(-\frac{\beta}{2}|\mathcal{C}|\right) \mathbb{E}^{\delta} \prod_{b \in \mathcal{C}} \exp \left[\frac{\beta}{4} \eta(b)^{2}\right] .
\end{aligned}
$$

For $\alpha \in(0, \beta / 4)$, we next define a new gradient Gibbs measure $\mu_{\alpha}$ (with expectation denoted $\left.\mathbb{E}_{\alpha}\right)$, such that $d \mu_{\alpha}=\left(Z_{\alpha}\right)^{-1} \exp \left(\alpha \sum_{b \in \mathcal{C}} \eta(b)^{2}\right) d \mu$, where $Z_{\alpha}$ is the corresponding partition function. In other words, for all $b \in\left(D^{\varepsilon}\right)^{*}$, the new pair potential is $V_{b}(x)=\beta V^{\delta}(x)-\alpha x^{2} 1_{\{b \in \mathcal{C}\}}$. Since $\alpha \in(0, \beta / 4)$, we have

$$
\inf _{b \in\left(D^{\varepsilon}\right)^{*}} \inf _{x \in \mathbb{R}} V_{b}^{\prime \prime}(x) \geq \beta \cos \delta-\alpha \geq \beta \cos \frac{\pi}{3}-\frac{\beta}{4}=\beta / 4 .
$$

Then by noting that $\mathbb{E}_{\alpha} \eta(b)=0$ for any $b \in \mathcal{C}$, and applying item 1 of Lemma 6 , with $c_{-} \geq \beta / 4$ by the last inequality, we obtain

$$
\begin{aligned}
\log \frac{Z_{\beta / 4}}{Z} & =\int_{0}^{\beta / 4} \sum_{b \in \mathcal{C}} \mathbb{E}_{\alpha}\left[\eta(b)^{2}\right] d \alpha \\
& =\int_{0}^{\beta / 4} \sum_{b \in \mathcal{C}} \operatorname{Var}_{\alpha}[\eta(b)] d \alpha \\
& \leq \int_{0}^{\beta / 4} \sum_{b \in \mathcal{C}} \frac{4}{\beta} d \alpha=|\mathcal{C}|,
\end{aligned}
$$

or

$$
\mathbb{E}^{\delta} \prod_{b \in \mathcal{C}} \exp \left[\frac{\beta}{4} \eta(b)^{2}\right]=\frac{Z_{\beta / 4}}{Z} \leq e^{|\mathcal{C}|} .
$$


Combining (11)-(13), we have

$$
\mathbb{E}^{\delta}\left[\prod_{(i, j) \in \mathcal{C}} 1_{|\theta(j)-\theta(i)|>a}\right] \leq\left(e \exp \left(-\beta a^{2} / \pi^{2}\right)\right)^{|\mathcal{C}|}
$$

which completes the proof.

In Sections 4 and 5 we will fix some $\delta \in(0, \pi / 3)$, and, abusing the notation when it is clear from the context, will use $V, \mu, \mathbb{E}$ to denote the corresponding convex potential, gradient Gibbs measure and expectation without indicating their dependence on $\delta$.

3.2. The Coupling. Let $\eta^{\delta}=\eta^{\delta, \varepsilon}$ denote the gradient variables associated with the Gibbs measure (9) on $D^{\varepsilon}$ (we will omit its dependence on the parameter $\varepsilon$ hereafter). Since $\eta^{\delta}$ is the gradient of a function, it satisfies the plaquette condition

$$
\eta^{\delta}\left(b_{12}\right)+\eta^{\delta}\left(b_{23}\right)+\eta^{\delta}\left(b_{34}\right)+\eta^{\delta}\left(b_{41}\right)=0,
$$

for any directed bonds $\left(b_{12}, b_{23}, b_{34}, b_{41}\right)$ that encloses a plaquette. More generally, $\eta^{\delta}$ is vortex-free. That is, for any given directed loop $\mathfrak{L} \subset D^{\varepsilon}$,

$$
\sum_{b \in \mathfrak{L}} \eta^{\delta}(b)=0 .
$$

Let $\eta$ denote the gradient variables associated with the XY model on $D^{\varepsilon}$, defined in (2). Since the spins are $S^{1}$-valued, when summing over any directed loop $\mathfrak{L}$, one has

$$
\sum_{b \in \mathfrak{L}} \eta(b)=2 k \pi, k \in \mathbb{Z}
$$

When $k \neq 0$, we say $\mathfrak{L}$ is a vortex for $\eta$.

In order to compare the scaling limits under the Gibbs meaures $\mu$ (defined in (1)) and $\mu^{\delta}$ (defined in (9)), we will introduce a coupling between the gradient variables $\eta$ and $\eta^{\delta}$, such that they all agree on an event with probability close to 1 . In Section 3.1 we assume that $\delta \in(0, \pi / 3]$, but for the purposes of the coupling in this section, $\delta$ can be anywhere in $(0, \pi / 2)$. Given some $\omega \in \mathbb{R}^{\left(D^{\varepsilon}\right)^{*}}$, we will say that an edge $b \in\left(D^{\varepsilon}\right)^{*}$ is $\delta$-bad (for that $\omega$ ), if $|\omega(b)|>\delta$; otherwise we say the edge is $\delta$-good. Let $\mathcal{B}$ denote the subset of $\omega$ 's such that there exists at least one $\delta$-bad edge (for that $\omega$ ) anywhere in $D^{\varepsilon}$. On $\mathcal{B}^{c}$, $|\eta(b)| \leq \delta$ for all $b \in\left(D^{\varepsilon}\right)^{*}$, and therefore for any directed bonds $\left(b_{12}, b_{23}, b_{34}, b_{41}\right)$ that encloses a plaquette,

$$
\left|\eta\left(b_{12}\right)+\eta\left(b_{23}\right)+\eta\left(b_{34}\right)+\eta\left(b_{41}\right)\right| \leq 4 \delta<2 \pi .
$$

Therefore, $\eta\left(b_{12}\right)+\eta\left(b_{23}\right)+\eta\left(b_{34}\right)+\eta\left(b_{41}\right)=0$. This implies that $\eta$ is vortex-free, and for any given loop $\mathfrak{L}$, (14) cannot hold unless $k=0$, because $D$ is discretely simply connected, as discussed in Section 2. Considering the $\eta^{\delta}$ field as also taking values in $\mathbb{R}^{\left(D^{\varepsilon}\right)^{*}}$, the same considerations apply to it. Thus $\eta$ and $\eta^{\delta}$ both satisfy the same curl-free constraints on $\mathcal{B}^{c}$.

Denote by $\left(\partial D^{\varepsilon}\right)^{*}$ the set of edges in $\left(D^{\varepsilon}\right)^{*}$ with both end vertices in $\partial D^{\varepsilon}$. Define

$$
\Omega=\left\{\omega:\left(D^{\varepsilon}\right)^{*} \rightarrow \mathbb{R}: \sum_{b \in \mathfrak{L}} \omega(b)=0 \text { for all loops } \mathfrak{L}, \text { and } \omega(b)=0 \text { for } b \in\left(\partial D^{\varepsilon}\right)^{*}\right\} .
$$

First notice that since $V^{\delta}(x) \geq-\cos x$, we have $Z^{\delta}=\mu^{\delta}(\Omega) Z^{\delta} \leq \mu(\Omega) Z \leq Z$. Let $\mathcal{F}$ be the $\sigma$-field generated by cylinder sets in $\mathbb{R}^{\left(D^{\varepsilon}\right)^{*}}$. We now introduce a coupling 
between $\eta$ and $\eta^{\delta}$ (thus also a coupling between $\tilde{\eta}$ and $\tilde{\eta}^{\delta}$ ) on a common probability space $\left(\mathbb{R}^{\left(D^{\varepsilon}\right)^{*}} \times \mathbb{R}^{\left(D^{\varepsilon}\right)^{*}}, \mathcal{F} \times \mathcal{F}\right)$, with marginals given by $\mu$ and $\mu^{\delta}$. Let

$$
\rho=\frac{\mu 1_{\mathcal{B}^{c}}}{\int 1_{\mathcal{B}^{c}} d \mu}=\frac{\mu^{\delta} 1_{\mathcal{B}^{c}}}{\int 1_{\mathcal{B}^{c}} d \mu^{\delta}},
$$

where the second equality follows from the fact that the unnormalized density of $\mu$ and $\mu^{\delta}$ coincide on $\mathcal{B}^{c}$ (by the definition of $V^{\delta}$ in (8) ). Also let

$$
\begin{aligned}
\nu & =\frac{\mu 1_{\mathcal{B}}}{\int 1_{\mathcal{B}} d \mu}, \nu^{\prime}=\frac{\mu^{\delta} 1_{\mathcal{B}}}{\int 1_{\mathcal{B}} d \mu^{\delta}}, \\
c & =\int 1_{\mathcal{B}^{c}} d \mu, c^{\prime}=\int 1_{\mathcal{B}^{c}} d \mu^{\delta} .
\end{aligned}
$$

Since on $\mathcal{B}^{c}$ we have $V^{\delta}(x)=-\cos x$, it follows that $c Z=c^{\prime} Z^{\delta}$, thus $c \leq c^{\prime}$ since $Z^{\delta} \leq Z$. We can therefore write

$$
\begin{aligned}
\mu & =c \rho+(1-c) \nu \\
\mu^{\delta} & =c^{\prime} \rho+\left(1-c^{\prime}\right) \nu^{\prime}=c \rho+(1-c) \lambda,
\end{aligned}
$$

where $\lambda=\left[\left(c^{\prime}-c\right) \rho+\left(1-c^{\prime}\right) \nu^{\prime}\right] /(1-c)$ is a probability measure. To describe the coupling, let $\eta, W$ be independent random variables taking values in $\mathbb{R}^{\left(D^{\varepsilon}\right)^{*}}$ with distributions $\mu$ and $\lambda$, respectively. Given $\eta$, if $\eta \notin \mathcal{B}$, set $\eta^{\delta}=\eta$; otherwise, if $\eta \in \mathcal{B}$, define $\eta^{\delta}=W$. It is straightforward to check that this $\eta^{\delta}$ has distribution $\mu^{\delta}$.

We now check that under this coupling, $\eta=\eta^{\delta}$ with high probability. Indeed,

$$
\mathbb{P}\left(\eta=\eta^{\delta}\right) \geq c=(1-\mu(\mathcal{B})) .
$$

Moreover, $\mathcal{B}$ occurs with small probability. Since by Theorem 5 , we have

$$
\mu(\mathcal{B}) \leq \sum_{b \in\left(D^{\varepsilon}\right)^{*}} \mu(|\eta(b)|>\delta) \leq 2 \varepsilon^{-d} c \exp \left(-\beta(\varepsilon) \delta^{2} / \pi^{2}\right) .
$$

For $\delta \in[\pi / 3, \pi / 2)$, the right hand side tends to zero as $\varepsilon \rightarrow 0$ because $\beta(\varepsilon)-9 d|\log \varepsilon| \rightarrow$ $\infty$. Thus under our coupling, $\mathbb{P}\left(\eta=\eta^{\delta}\right) \rightarrow 1$ as $\varepsilon \rightarrow 0$.

Remark 8. (15) and our earlier discussion show that if $\beta(\varepsilon)-9 d|\log \varepsilon| \rightarrow \infty$, then with probability close to 1 , for small $\varepsilon$ the model is vortex free.

\section{Fludtuations of GRADiEnt FiELdS ON $\mathbb{Z}^{d}$}

In this section we prove the GFF scaling limit for the rescaled gradient variable asscociated with the gradient Gibbs measure on $\mathbb{Z}^{d}$. As noted at the end of Section 3.1 above, henceforth we use a simplified notation that does not specify the cutoff parameter $\delta \in(0, \pi / 3)$ needed to make $V^{\delta}$ (now denoted $V$ ) strictly convex in $\mathbb{R}$. Let $\eta^{\varepsilon} \in \mathbb{R}^{\left(\varepsilon \mathbb{Z}^{d}\right)^{*}}$ be sampled from the gradient Gibbs measure, defined formally as

$$
d \mu^{\varepsilon}=Z^{-1} \exp \left[-\beta(\varepsilon) \sum_{b \in\left(\varepsilon \mathbb{Z}^{d}\right)^{*}} V\left(\eta^{\varepsilon}(b)\right)\right] \prod_{i \in \varepsilon \mathbb{Z}^{d}} d \theta^{\varepsilon}(i),
$$

such that $\eta^{\varepsilon}(b)=\theta^{\varepsilon}(j)-\theta^{\varepsilon}(i)$ if $b=(i, j)$, and the potential $V: \mathbb{R} \rightarrow \mathbb{R}$ satisfies

$$
0<c_{-} \leq V^{\prime \prime}(x) \leq c_{+}<\infty, V^{\prime \prime} \text { is Lipschitz continuous, and } V^{\prime \prime}(0)=1 .
$$

Although it is not a priori clear that the infinite volume Gibbs measure (16) is well defined, in [FS97] the existence and uniqueness was proved for the translation invariant 
and ergodic Gibbs states of (16), with any fixed slope (i.e., the macroscopic tilt of the $\theta^{\varepsilon}$ field). In what follows we focus on the (unique) zero tilt Gibbs state of (16), which arises as the infinite volume limit of the zero boundary condition Gibbs measure (7).

Given $\varepsilon>0$, define the rescaled gradient variable $\tilde{\eta}^{\varepsilon}=\sqrt{\beta(\varepsilon)} \eta^{\varepsilon}$. Notice that the law of $\tilde{\eta}^{\varepsilon}$ is given by $\tilde{Z}^{-1} \exp \left(-\tilde{H}\left(\tilde{\eta}^{\varepsilon}\right)\right) \prod_{i \in \varepsilon \mathbb{Z}^{d}} d \tilde{\theta}^{\varepsilon}(i)$, with Hamiltonian $\tilde{H}\left(\tilde{\eta}^{\varepsilon}\right)=\sum_{b \in\left(\varepsilon \mathbb{Z}^{d}\right)^{*}} \tilde{V}\left(\tilde{\eta}^{\varepsilon}(b)\right)$, where

$$
\tilde{V}(x)=\tilde{V}_{\varepsilon}(x)=\beta(\varepsilon) V(x / \sqrt{\beta(\varepsilon)}),
$$

and thus $\tilde{V}^{\prime \prime}(x)=V^{\prime \prime}(x / \sqrt{\beta})$. Moreover,

$$
0<c_{-} \leq \inf _{\varepsilon>0} \inf _{x \in \mathbb{R}} \tilde{V}^{\prime \prime}(x) \leq \sup _{\varepsilon>0} \sup _{x \in \mathbb{R}} \tilde{V}^{\prime \prime}(x) \leq c_{+}<\infty .
$$

For test functions $\varphi \in \mathcal{C}_{0}^{\infty}\left(\mathbb{R}^{d}\right)$, we define the fluctuation field

$$
\left\langle\tilde{\eta}^{\varepsilon}, \varphi\right\rangle=\varepsilon^{d / 2-1} \sum_{b \in\left(\varepsilon \mathbb{Z}^{d}\right)^{*}} \nabla \varphi(b) \tilde{\eta}^{\varepsilon}(b) .
$$

We now state the following CLT (central limit theorem) type result.

Theorem 9. Suppose that the potential $V: \mathbb{R} \rightarrow \mathbb{R}$ satisfies 17 , and $\beta(\varepsilon) \rightarrow \infty$ as $\varepsilon \rightarrow 0$. Then for all $t \in \mathbb{R}$, and $\varphi \in C_{0}^{\infty}\left(\mathbb{R}^{d}\right)$,

$$
\lim _{\varepsilon \rightarrow 0} \mathbb{E}\left[e^{t\left\langle\tilde{\eta}^{\varepsilon}, \varphi\right\rangle}\right]=\exp \left[\frac{t^{2}}{2}\langle\partial \varphi, \partial \varphi\rangle\right]
$$

where $\langle\partial \varphi, \partial \varphi\rangle$ is defined in (6).

Notice that when $\beta$ does not depend on $\varepsilon$, the analogue of Theorem 9 was proved in [NS97, with the quadratic form of (6) replaced by the inverse of an elliptic operator (depending on $\tilde{V}$ ). We will prove Theorem 9 by adapting the homogenization argument in GOS01 (which was a probabilistic version of the argument of [NS97]) to the case where $\beta=\beta(\varepsilon)$ and $\tilde{V}$ depends on $\varepsilon$.

4.1. The random walk representation. The study of scaling limits of the gradient Gibbs measure (16) is usually based (see [HS94], [DGI00], GOS01]) on a representation of the covariance of local functions of the field in terms of a random walk in random conductances, known as the Helffer-Sjöstrand representation.

We now introduce this random walk representation, in the simple case where $\varepsilon=\beta=1$. It adapts to the general case (where $\beta=\beta(\varepsilon)$, and the random walk is on $\varepsilon \mathbb{Z}^{d}$ ) in a straightforward manner. Consider a Langevin dynamics for $\theta$, consisting of the system of SDEs,

$$
d \theta_{t}(x)=-\sum_{b \ni x} V^{\prime}\left(\eta_{t}(b)\right) d t+\sqrt{2} d B_{x}(t), x \in \mathbb{Z}^{d},
$$

where $\left\{B_{x}(t), x \in \mathbb{Z}^{d}\right\}$ is a collection of independent standard Brownian motions. For $b=(x, y) \in\left(\mathbb{Z}^{d}\right)^{*}$, set $\eta_{t}(b)=\theta_{t}(y)-\theta_{t}(x)$. This Langevin dynamics is reversible with respect to the gradient Gibbs measure (16).

The Langevin dyamics induces a Markov dynamics on the gradient field $\eta$. We first introduce some notation before describing its generator. For $\alpha=1, \ldots, d$, the difference operator $\nabla_{\alpha}$ acting on functions $f: \mathbb{Z}^{d} \rightarrow \mathbb{R}$ is simply $\nabla_{\alpha} f(x)=f\left(x+e_{\alpha}\right)-f(x)$. Its adjoint operator $\nabla_{\alpha}^{*}$ is then $\left(\nabla_{\alpha}^{*} f\right)(x)=f(x)-f\left(x-e_{\alpha}\right)$. The generator is given by

$$
L F(\eta)=-\sum_{x \in \mathbb{Z}^{d}}\left[\partial_{x}^{2} F(\eta)-\left(\sum_{\alpha=1}^{d} \nabla_{\alpha}^{*} V^{\prime}\left(\eta\left(x, x+e_{\alpha}\right)\right)\right) \partial_{x} F(\eta)\right],
$$


where $F$ is a smooth local function on $\mathbb{R}^{\left(\mathbb{Z}^{d}\right)^{*}}$, and $\partial_{x}=\frac{\partial}{\partial \theta(x)}$.

A basic object for probabilistic study of a gradient field model is a random walk $X(t)$ on $\mathbb{Z}^{2}$ under the dynamic random environment $\eta_{t}$. Given the process (19) with initial condition $\eta_{0},\{X(t)\}_{t>0}$ is the random walk that performs nearest neighbor jumps starting from $X(0)=x$, with time dependent jump rate $V^{\prime \prime}\left(\eta_{t}(b)\right)$ along the directed edge $b$. Since $V$ is uniformly convex, the random environment is uniformly elliptic. The combined generator for $\eta_{t}$ and $X(t)$ is given by $\mathcal{L}=L+Q$, where $Q$ is defined by

$$
Q F(x, \eta)=-\sum_{\alpha=1}^{d} \nabla_{\alpha}^{*} V^{\prime \prime}\left(\eta\left(x, x+e_{\alpha}\right)\right) \nabla_{\alpha} F(x, \eta),
$$

for any function $F$ that has compact support in $x$, and is a smooth local function of $\eta$. Denote by $\mathbb{E}_{x, \eta}$ the quenched expectation with respect to the law of $X(t)$, given the environment process $\left\{\eta_{t}\right\}_{t \geq 0}$.

We now state the main result for the covariance representation, established in GOS01. We set $\partial F(x, \eta)=\partial_{x} F(\eta)$.

Proposition 10 (GOS01]). For any twice continuously differentiable and square integrable local functions $F, G$, such that both $L F$ and $L G$ are $L^{2}$ integrable,

$$
\mathbb{E}[F(\eta) G(\eta)]-\mathbb{E}[F(\eta)] \mathbb{E}[G(\eta)]=\int_{0}^{\infty} \sum_{x \in \mathbb{Z}^{d}} \mathbb{E}\left[\partial F(x, \eta) \mathbb{E}_{x, \eta}[\partial G(X(t), \eta(t))]\right] d t,
$$

where $\mathbb{E}$ is the expectation with respect to the Gibbs measure (16).

4.2. An averaged CLT. To apply Proposition 10, we replace $\mathbb{Z}^{d}, \eta, V$ by $\varepsilon \mathbb{Z}^{d}, \tilde{\eta}^{\varepsilon}, \tilde{V}$ respectively. The main result in this section is the following.

Proposition 11. As $\varepsilon \rightarrow 0, \varepsilon X^{\varepsilon}\left(t \varepsilon^{-2}\right)$ converges weakly to the standard Brownian motion in the Skorohod topology $D\left([0, T]: \mathbb{R}^{d}\right)$, for any $T \geq 0$.

The proof of Proposition 11 is an adaptation of the argument in Section 4 of [GOS01, which applies the Kipnis-Varadhan method [KV86]. As we pointed out before, the main difference between the setup in GOS01 and this paper is that in our setting, the potential $\tilde{V}$ (and thus the gradient Gibbs measure $\mu^{\varepsilon}$ ) changes with $\varepsilon$ because $\beta=\beta(\varepsilon)$. However, thanks to the contour estimate (Proposition 1) and the stationarity of the process $\eta_{t}$ in $t$, at any given time $t$, the probability to have a large $\left|\eta_{t}^{\varepsilon}(b)\right|$ for any edge $b$ is small. Therefore, at any time $t$ the jump rates of $X^{\varepsilon}$ concentrate around $V^{\prime \prime}(0)$. We will use this fact to prove an (averaged) invariance principle for $X^{\varepsilon}(t)$.

Let $\tau_{x}, x \in \varepsilon \mathbb{Z}^{d}$ denote the shift by $x$ : for $\eta \in \mathbb{R}^{\left(\varepsilon \mathbb{Z}^{d}\right)^{*}}, \tau_{x} \eta(b)=\eta(b+x)$. For $x=e_{\alpha}$, $\alpha=1, \ldots, d$, we write $\tau_{\alpha}$ for $\tau_{e_{\alpha}}$. Also, for any smooth local function $F$ on $\mathbb{R}^{\left(\varepsilon \mathbb{Z}^{d}\right)^{*}}$, define $D_{\alpha} F(\eta)=F\left(\tau_{\alpha} \eta\right)-F(\eta)$, and denote by $D_{\alpha}^{*}$ the adjoint operator of $D_{\alpha}$. Consider the process that describes the environment seen from the random walk

$$
\hat{\eta}_{t}^{\varepsilon}=\tau_{-X \varepsilon}^{\varepsilon}(t) \tilde{\eta}_{t}^{\varepsilon}
$$

Notice that $\hat{\eta}^{\varepsilon}$ is a stationary process in $t$. As was discussed in GOS01, its generator is given by a self adjoint operator

$$
\tilde{\mathcal{L}} F(\eta)=L F(\eta)+\sum_{\alpha=1}^{d} D_{\alpha}^{*}\left[\tilde{V}^{\prime \prime}\left(\eta\left(0, \varepsilon e_{\alpha}\right)\right) D_{\alpha} F\right](\eta)
$$

We denote by $\tilde{\mathbb{P}}$ and $\tilde{\mathbb{E}}$ the law and corresponding expectation of the process $\hat{\eta}_{t}^{\varepsilon}$. By Lemma 4.2 of GOS01, $\hat{\eta}^{\varepsilon}$ is also time ergodic. 
For the proof below, we will switch between two measures: $\tilde{\mathbb{P}}$ (and $\tilde{\mathbb{E}}$ ) that characterizes the law of the environment seen from the random walk $\hat{\eta}_{\text {. }}^{\varepsilon}$, and $\mathbb{P}$ (and $\mathbb{E}$ ) that characterizes the stationary measure of $\eta^{\varepsilon}$. (i.e., the gradient Gibbs measure (16)).

Denote by $\mathcal{F}$ the natural filtration generated by $\left\{\hat{\eta}_{s}^{\varepsilon}\right\}_{s>0}$. Observe that $X^{\varepsilon}$ can be decomposed as an additive functional of the process $\hat{\eta}^{\varepsilon}$ plus a martingale with bounded increments: for $\alpha=1, \ldots, d$,

$$
X_{\alpha}^{\varepsilon}(t)=M_{\alpha}^{\varepsilon}(t)+\int_{0}^{t}\left(D_{\alpha}^{*} \tilde{V}^{\prime \prime}\right)\left(\hat{\eta}_{s}^{\varepsilon}\left(0, \varepsilon e_{\alpha}\right)\right) d s,
$$

where $M_{\alpha}^{\varepsilon}$ is a $(\tilde{\mathbb{P}}, \mathcal{F})$ martingale, such that

$$
\tilde{\mathbb{E}}\left[M_{\alpha}^{\varepsilon}(t) M_{\beta}^{\varepsilon}(t)\right]=2 t \tilde{\mathbb{E}} \tilde{V}^{\prime \prime}\left(\hat{\eta}_{t}^{\varepsilon}\left(0, \varepsilon e_{\alpha}\right)\right) \delta_{\alpha \beta} .
$$

We now claim $\tilde{\mathbb{E}} \tilde{V}^{\prime \prime}\left(\hat{\eta}_{t}^{\varepsilon}\left(0, \varepsilon e_{\alpha}\right)\right) \rightarrow 1$ as $\varepsilon \rightarrow 0$. Since $\hat{\eta}^{\varepsilon}$ is stationary, and for any $t, \eta_{t}^{\varepsilon}$ is distributed according to the translation invariant Gibbs measure (16), by Proposition 7. there exists $c<\infty$, such that

$$
\mathbb{P}\left(\left|\eta_{t}^{\varepsilon}\left(0, \varepsilon e_{\alpha}\right)\right|>\beta^{-1 / 3}\right) \leq c \exp \left(-\beta^{1 / 3} / \pi^{2}\right) .
$$

On the other hand, when $\left|\eta_{t}^{\varepsilon}\left(0, \varepsilon e_{\alpha}\right)\right| \leq \beta^{-1 / 3}$, by the Lipschitz continuity of $V^{\prime \prime}$, there exists $C<\infty$, such that $\left|V^{\prime \prime}\left(\eta_{t}^{\varepsilon}\left(0, \varepsilon e_{\alpha}\right)\right)-1\right| \leq C \beta^{-1 / 3}$. We have, by decomposing the space into the event on the left hand side of (22) and its complement and using the Lipschitz continuity of $V^{\prime \prime}$,

$$
\begin{aligned}
\left|\tilde{\mathbb{E}} \tilde{V}^{\prime \prime}\left(\hat{\eta}_{t}^{\varepsilon}\left(0, \varepsilon e_{\alpha}\right)\right)-1\right| & =\left|\mathbb{E} V^{\prime \prime}\left(\eta_{t}^{\varepsilon}\left(0, \varepsilon e_{\alpha}\right)\right)-1\right| \\
& \leq\left(1+\sup _{x \in \mathbb{R}} V^{\prime \prime}(x)\right) c \exp \left(-\beta^{1 / 3} / \pi^{2}\right)+C \beta^{-1 / 3} .
\end{aligned}
$$

Since $\beta \rightarrow \infty$ as $\varepsilon \rightarrow 0$, we conclude that $\left|\tilde{\mathbb{E}} \tilde{V}^{\prime \prime}\left(\hat{\eta}_{t}^{\varepsilon}\left(0, \varepsilon e_{\alpha}\right)\right)-1\right| \rightarrow 0$. Therefore for any $t>0$,

$$
\tilde{\mathbb{E}}\left[\left|M_{\alpha}^{\varepsilon}(t)\right|^{2}\right] \rightarrow 2 t \text { as } \varepsilon \rightarrow 0 .
$$

By a version of the martingale functional central limit theorem (see for example [Hel82]), $\varepsilon M_{\alpha}^{\varepsilon}\left(\varepsilon^{-2} \cdot\right) \rightarrow B$. in the Skorohod topology.

For the second term in (20), first notice that if $\beta$ is independent of $\varepsilon$ (thus $\tilde{V}^{\prime \prime}$ is a fixed function independent of $\varepsilon$ ), a central limit theorem for $\varepsilon \int_{0}^{t \varepsilon^{-2}}\left(D_{\alpha}^{*} \tilde{V}^{\prime \prime}\right)\left(\hat{\eta}_{s}^{\varepsilon}\left(0, \varepsilon e_{\alpha}\right)\right) d s$ follows from Theorem 1.8 and Corollary 1.9 of [KV86. Here, because of the dependence of $\tilde{V}^{\prime \prime}$ on $\varepsilon$, we need a triangular array central limit theorem. However, an analog of Theorem 1.8 of [KV86] still holds, stated as Lemma 12 below. We apply Lemma 12 with $\hat{\eta}^{\varepsilon}=\hat{\eta}^{\varepsilon}\left(0, \varepsilon e_{\alpha}\right)$, and $V^{\varepsilon}=D_{\alpha}^{*} \tilde{V}^{\prime \prime}$. Condition (27) of the lemma is verified by the same argument as (4.10) of GOS01, with the constant $C$ only depending on $c_{-}$in (18). It follows that there is for each $\alpha$ another square integrable martingale $\mathcal{M}_{\alpha}^{\varepsilon}(\cdot)$, with stationary increments, such that if we define for $s>0$ and $\alpha=1, \ldots, d$,

$$
e_{\alpha}^{\varepsilon}(s)=\int_{0}^{s}\left(D_{\alpha}^{*} \tilde{V}^{\prime \prime}\right)\left(\hat{\eta}_{u}^{\varepsilon}\left(0, \varepsilon e_{\alpha}\right)\right) d u-\mathcal{M}_{\alpha}^{\varepsilon}(s),
$$

then for any $t>0$,

$$
\lim _{\varepsilon \rightarrow 0} \varepsilon \sup _{s \in\left[0, \varepsilon^{-2} t\right]}\left|e_{\alpha}^{\varepsilon}(s)\right|=0 \text { in probability, }
$$


and moreover, the covariance of the increment of $\mathcal{M}_{\alpha}^{\varepsilon}(s)$ satisfies

$$
\begin{aligned}
& \tilde{\mathbb{E}}\left[\mathcal{M}_{\alpha}^{\varepsilon}(t+1)-\mathcal{M}_{\alpha}^{\varepsilon}(t)\right]\left[\mathcal{M}_{\beta}^{\varepsilon}(t+1)-\mathcal{M}_{\beta}^{\varepsilon}(t)\right] \\
= & 2 \int_{0}^{\infty} \tilde{\mathbb{E}}\left[\left(D_{\alpha}^{*} \tilde{V}^{\prime \prime}\right)\left(\hat{\eta}_{0}^{\varepsilon}\left(0, \varepsilon e_{\alpha}\right)\right)\left(D_{\beta}^{*} \tilde{V}^{\prime \prime}\right)\left(\hat{\eta}_{t}^{\varepsilon}\left(0, \varepsilon e_{\beta}\right)\right)\right] d t .
\end{aligned}
$$

By (20) and (24), it suffices to prove a martingale central limit theorem for $\varepsilon\left(M^{\varepsilon}\left(\varepsilon^{-2} t\right)+\right.$ $\left.\mathcal{M}^{\varepsilon}\left(\varepsilon^{-2} t\right)\right)$. To do that we will make use of the fact that this process has uniformly bounded increments (independent of $\varepsilon$ ). For fixed $\varepsilon>0$, combining (21), (25)), and (4.11)-(4.12) of [GOS01], we see that the increment of $M^{\varepsilon}+\mathcal{M}^{\varepsilon}$ from $t$ to $t+1$ has the quadratic form

$$
q_{\alpha \beta}^{\varepsilon}=2 \tilde{\mathbb{E}} \tilde{V}^{\prime \prime}\left(\hat{\eta}_{0}^{\varepsilon}\left(0, \varepsilon e_{\alpha}\right)\right) \delta_{\alpha \beta}-2 \int_{0}^{\infty} \tilde{\mathbb{E}}\left[\left(D_{\alpha}^{*} \tilde{V}^{\prime \prime}\right)\left(\hat{\eta}_{0}^{\varepsilon}\left(0, \varepsilon e_{\alpha}\right)\right)\left(D_{\beta}^{*} \tilde{V}^{\prime \prime}\right)\left(\hat{\eta}_{t}^{\varepsilon}\left(0, \varepsilon e_{\beta}\right)\right)\right] d t .
$$

This quantity can be characterized through a variational formula on $\varepsilon \mathbb{Z}^{d}$ (see, e.g. (2.12) of [GOS01]). Thus, arguing as in GOS01, we obtain the following analogue of (4.13) of GOS01]: for any $v \in \mathbb{R}^{d}$,

$$
2 \sum_{\alpha=1}^{d} v_{\alpha}^{2}\left[\tilde{\mathbb{E}}\left(\left[\tilde{V}^{\prime \prime}\left(\hat{\eta}_{0}^{\varepsilon}\left(0, \varepsilon e_{\alpha}\right)\right)\right]^{-1}\right)\right]^{-1} \leq v \cdot q^{\varepsilon} v \leq 2 \sum_{\alpha=1}^{d} v_{\alpha}^{2} \tilde{\mathbb{E}} \tilde{V}^{\prime \prime}\left(\hat{\eta}_{0}^{\varepsilon}\left(0, \varepsilon e_{\alpha}\right)\right) .
$$

We will prove that as $\varepsilon \rightarrow 0$,

$$
\tilde{\mathbb{E}} \tilde{V}^{\prime \prime}\left(\hat{\eta}_{0}^{\varepsilon}\left(0, \varepsilon e_{\alpha}\right)\right) \rightarrow 1, \tilde{\mathbb{E}}\left(\left[\tilde{V}^{\prime \prime}\left(\hat{\eta}_{0}^{\varepsilon}\left(0, \varepsilon e_{\alpha}\right)\right)\right]^{-1}\right) \rightarrow 1,
$$

so that

$$
v \cdot q^{\varepsilon} v \rightarrow 2 \sum_{\alpha=1}^{d} v_{\alpha}^{2}
$$

Therefore the conclusion of the proposition follows from a version of the functional central limit theorem for martingales with uniformly bounded increments (see e.g. [Hel82]).

We prove (26) by applying Proposition 7 to show that as $\varepsilon \rightarrow 0$, the value of $\tilde{V}^{\prime \prime}\left(\hat{\eta}_{0}^{\varepsilon}\left(0, \varepsilon e_{\alpha}\right)\right)$ highly concentrates around 1 . Indeed, $\tilde{\mathbb{E}} \tilde{V}^{\prime \prime}\left(\hat{\eta}_{0}^{\varepsilon}\left(0, \varepsilon e_{\alpha}\right)\right) \rightarrow 1$ follows from (23) ilar argument using (22) yields

$$
\begin{aligned}
\tilde{\mathbb{E}}\left|\left[\tilde{V}^{\prime \prime}\left(\hat{\eta}_{0}^{\varepsilon}\left(0, \varepsilon e_{\alpha}\right)\right)\right]^{-1}-1\right| \leq & \max \left\{1-\left(1+C^{\prime} \beta^{-1 / 3}\right)^{-1},\left(1-C^{\prime} \beta^{-1 / 3}\right)^{-1}-1\right\} \\
& +\left(1+\left(\inf _{x \in \mathbb{R}} V^{\prime \prime}(x)\right)^{-1}\right) c \exp \left(-\beta^{1 / 3} / \pi^{2}\right),
\end{aligned}
$$

for some $C^{\prime}<\infty$. Since $\beta(\varepsilon) \rightarrow \infty$, the right hand side above tends to zero as $\varepsilon \rightarrow 0$.

Lemma 12. Let $\hat{\eta}^{\varepsilon}$ be the Markov process with generator $\tilde{\mathcal{L}}$, defined above following the statement of Proposition [1]. Let $V^{\varepsilon}$ for each $\varepsilon$ be a function satisfying $\tilde{\mathbb{E}} V^{\varepsilon}\left(\hat{\eta}_{0}^{\varepsilon}\right)=0$, and such that there is a constant $C$ (not depending on $\varepsilon$ ), so that

$$
\tilde{\mathbb{E}}\left[V^{\varepsilon}\left(\hat{\eta}_{0}^{\varepsilon}\right) \phi\left(\hat{\eta}_{0}^{\varepsilon}\right)\right] \leq C \tilde{\mathbb{E}}\left[\phi\left(\hat{\eta}_{0}^{\varepsilon}\right) \tilde{\mathcal{L}} \phi\left(\hat{\eta}_{0}^{\varepsilon}\right)\right]^{1 / 2}, \text { for all } \phi \in \mathcal{D}(\tilde{\mathcal{L}}) \text {. }
$$

Define

$$
Y^{\varepsilon}(t)=\int_{0}^{t} V^{\varepsilon}\left(\hat{\eta}_{s}^{\varepsilon}\right) d s
$$


Then there exists a square integrable martingale $\mathcal{M}^{\varepsilon}(\cdot)$ with stationary increments, such that for any $t>0$,

$$
\lim _{\varepsilon \rightarrow 0} \sup _{s \in\left[0, \varepsilon^{-2} t\right]} \varepsilon\left|Y^{\varepsilon}(s)-\mathcal{M}^{\varepsilon}(s)\right|=0 \text { in probability. }
$$

Moreover,

$$
\begin{aligned}
\tilde{\mathbb{E}}\left|\mathcal{M}^{\varepsilon}(t+1)-\mathcal{M}^{\varepsilon}(t)\right|^{2} & =-2 \tilde{\mathbb{E}}\left[V^{\varepsilon}\left(\hat{\eta}_{0}^{\varepsilon}\right) \tilde{\mathcal{L}}^{-1} V^{\varepsilon}\left(\hat{\eta}_{0}^{\varepsilon}\right)\right] \\
& =2 \int_{0}^{\infty} \tilde{\mathbb{E}}\left[V^{\varepsilon}\left(\hat{\eta}_{0}^{\varepsilon}\right) V^{\varepsilon}\left(\hat{\eta}_{t}^{\varepsilon}\right)\right] d t .
\end{aligned}
$$

In the case of a discrete time Markov chain we have the following analogous statement. Let $\left\{\eta_{j}^{\varepsilon}\right\}$ be a Markov chain with transition operator $q^{\varepsilon}$, reversible with stationary distribution $\mu=\mu^{\varepsilon}$ (and denote by $\mathbb{E}$ the corresponding expectation). Let $V^{\varepsilon}$ for each $\varepsilon$ be a function satisfying $\mathbb{E} V^{\varepsilon}\left(\eta_{0}^{\varepsilon}\right)=0$ and such that there is an absolute constant $C$, such that

$$
\mathbb{E}\left[V^{\varepsilon}\left(\eta_{0}^{\varepsilon}\right) \phi\left(\eta_{0}^{\varepsilon}\right)\right] \leq C \mathbb{E}\left[\phi\left(\eta_{0}^{\varepsilon}\right)\left(I-q^{\varepsilon}\right) \phi\left(\eta_{0}^{\varepsilon}\right)\right]^{1 / 2}, \text { for all } \phi \in L^{2}(\mu)
$$

Define

$$
Y_{n}^{\varepsilon}=\sum_{j=1}^{n} V^{\varepsilon}\left(\eta_{j}^{\varepsilon}\right) .
$$

Then there exists a square integrable martingale $\left\{\mathcal{M}_{j}^{\varepsilon}\right\}$ with stationary increments, such that for any $t>0$,

$$
\lim _{\varepsilon \rightarrow 0} \sup _{1 \leq j \leq\left[\varepsilon^{-2} t\right]} \varepsilon\left|Y_{j}^{\varepsilon}-\mathcal{M}_{j}^{\varepsilon}\right|=0 \text { in probability. }
$$

Moreover,

$$
\mathbb{E}\left|\mathcal{M}_{n}^{\varepsilon}-\mathcal{M}_{n-1}^{\varepsilon}\right|^{2}=\mathbb{E}\left[V^{\varepsilon}\left(\eta_{0}^{\varepsilon}\right)\left(I+q^{\varepsilon}\right)\left(I-q^{\varepsilon}\right)^{-1} V^{\varepsilon}\left(\eta_{0}^{\varepsilon}\right)\right] .
$$

Proof. If we replace $V^{\varepsilon}$ by a function $V$ that does not depend on $\varepsilon$, Lemma 12 is just a restatement of Theorems 1.3 and 1.8 (for the fixed lattice $\mathbb{Z}^{d}$ ) of [KV86. Indeed, for fixed $\varepsilon>0$, the constructions of the martingales in Theorems 1.3 and 1.8 of [KV86] yield (29) and (32). Therefore it suffices to check (28) and (31). As was discussed in [KV86, since the continuous time processes $Y^{\varepsilon}(\cdot)$ and $\mathcal{M}^{\varepsilon}(\cdot)$ are a.s. right continous, (28) will follow from the discrete time (31) after replacing the supremum in $\left[0, \varepsilon^{-2} t\right]$ by a supremum over dyadic times. Therefore we will only sketch the key idea to prove (31).

The main difference between (31) and Theorem 1.3 of [KV86] is that we need to bound a triangular array $\varepsilon\left(Y_{j}^{\varepsilon}-\mathcal{M}_{j}^{\varepsilon}\right)$. However, the proof of that theorem adapts to the current setting because (30) gives control of $V^{\varepsilon}$ uniformly in $\varepsilon$. As in that theorem, we begin by defining for all $\varepsilon, \delta>0$,

$$
u^{\varepsilon, \delta}=\left(I-q^{\varepsilon}+\delta\right)^{-1} V^{\varepsilon},
$$

and two sequences

$$
\begin{aligned}
\mathcal{M}_{n}^{\varepsilon, \delta} & =\sum_{j=0}^{n-1}\left[u^{\varepsilon, \delta}\left(\eta_{j+1}^{\varepsilon}\right)-q^{\varepsilon} u^{\varepsilon, \delta}\left(\eta_{j}^{\varepsilon}\right)\right] \\
& =\sum_{j=0}^{n-1}\left[u^{\varepsilon, \delta}\left(\eta_{j+1}^{\varepsilon}\right)-u^{\varepsilon, \delta}\left(\eta_{j}^{\varepsilon}\right)+V^{\varepsilon}\left(\eta_{j}^{\varepsilon}\right)\right], \\
e_{n}^{\varepsilon, \delta} & =u^{\varepsilon, \delta}\left(\eta_{0}^{\varepsilon}\right)-u^{\varepsilon, \delta}\left(\eta_{n}^{\varepsilon}\right) .
\end{aligned}
$$


Then, using the upper bound (30) for fixed $\varepsilon>0$, the same argument as in the proof of Theorem 1.3 of [KV86] implies the $L^{2}$ limits

$$
\lim _{\delta \rightarrow 0} \mathcal{M}_{n}^{\varepsilon, \delta} \doteq \mathcal{M}_{n}^{\varepsilon}, \quad \lim _{\delta \rightarrow 0} e_{n}^{\varepsilon, \delta} \doteq e_{n}^{\varepsilon},
$$

such that $Y_{n}^{\varepsilon}=\mathcal{M}_{n}^{\varepsilon}+e_{n}^{\varepsilon}$. Moreover, the uniform bound (30) implies the following analogs of $(1.11)$ - (1.12) of [KV86] along a triagular array:

$$
\begin{aligned}
\lim _{\varepsilon \rightarrow 0} \mathbb{E}\left|\mathcal{M}_{1}^{\varepsilon, \varepsilon^{2}}-\mathcal{M}_{1}^{\varepsilon}\right|^{2} & =0, \\
\lim _{\varepsilon_{0}, \varepsilon \rightarrow 0}\left\langle u^{\varepsilon, \varepsilon_{0}}-u^{\varepsilon, \varepsilon^{2}},\left(I-q^{\varepsilon}\right)\left(u^{\varepsilon, \varepsilon_{0}}-u^{\varepsilon, \varepsilon^{2}}\right)\right\rangle & =0, \\
\lim _{\varepsilon \rightarrow 0} \varepsilon^{2}\left\langle u^{\varepsilon, \varepsilon^{2}}, u^{\varepsilon, \varepsilon^{2}}\right\rangle & =0 .
\end{aligned}
$$

To prove (31), we write, as in [KV86],

$$
e_{j}^{\varepsilon}=\left(\mathcal{M}_{j}^{\varepsilon, \varepsilon^{2}}-\mathcal{M}_{j}^{\varepsilon}\right)+e_{j}^{\varepsilon, \varepsilon^{2}}+\varepsilon^{2} \sum_{k=0}^{j-1} u^{\varepsilon, \varepsilon^{2}}\left(\eta_{k}^{\varepsilon}\right) .
$$

It suffices to bound the tail probability for each term. Fix $\alpha>0$. By Doob's inequality and (33),

$$
\begin{aligned}
\mathbb{P}\left(\sup _{1 \leq j \leq\left[\varepsilon^{-2}\right]}\left|\mathcal{M}_{j}^{\varepsilon, \varepsilon^{2}}-\mathcal{M}_{j}^{\varepsilon}\right|>\alpha \varepsilon^{-1}\right) & \leq \frac{\varepsilon^{2}}{\alpha^{2}} \mathbb{E}\left|\mathcal{M}_{\left[\varepsilon^{-2}\right]}^{\varepsilon, \varepsilon^{2}}-\mathcal{M}_{\left[\varepsilon^{-2}\right]}^{\varepsilon}\right|^{2} \\
& \leq \frac{1}{\alpha^{2}} \mathbb{E}\left|\mathcal{M}_{1}^{\varepsilon, \varepsilon^{2}}-\mathcal{M}_{1}^{\varepsilon}\right|^{2} \\
& \rightarrow 0 \text { as } \varepsilon \rightarrow 0 .
\end{aligned}
$$

By (35),

$$
\begin{aligned}
\mathbb{P}\left(\sup _{\left.1 \leq j \leq \varepsilon^{-2}\right]}\left|\varepsilon^{2} \sum_{k=0}^{j-1} u^{\varepsilon, \varepsilon^{2}}\left(\eta_{k}^{\varepsilon}\right)\right|>\alpha \varepsilon^{-1}\right) & \leq \frac{\varepsilon^{2}}{\alpha^{2}} \mathbb{E}\left|\varepsilon^{2} \sum_{j=0}^{\left[\varepsilon^{-2}\right]-1}\right| u^{\varepsilon, \varepsilon^{2}}\left(\eta_{j}^{\varepsilon}\right)||^{2} \\
& \leq \frac{\varepsilon^{2}}{\alpha^{2}}\left\langle u^{\varepsilon, \varepsilon^{2}}, u^{\varepsilon, \varepsilon^{2}}\right\rangle \\
& \rightarrow 0 \text { as } \varepsilon \rightarrow 0 .
\end{aligned}
$$

Finally, for fixed $\varepsilon_{0}>0$, we write

$$
e_{j}^{\varepsilon, \varepsilon^{2}}=u^{\varepsilon, \varepsilon^{2}}\left(\eta_{0}^{\varepsilon}\right)-u^{\varepsilon, \varepsilon^{2}}\left(\eta_{j}^{\varepsilon}\right)=u^{\varepsilon, \varepsilon^{2}}\left(\eta_{0}^{\varepsilon}\right)-u^{\varepsilon, \varepsilon_{0}}\left(\eta_{j}^{\varepsilon}\right)+\left(u^{\varepsilon, \varepsilon_{0}}\left(\eta_{j}^{\varepsilon}\right)-u^{\varepsilon, \varepsilon^{2}}\left(\eta_{j}^{\varepsilon}\right)\right) .
$$

The supremum over the first two terms on the right hand side above can be bounded similarly to (36). The last term can be bounded by applying the elementary Lemma 1.4 of [KV86] and (34). This yields (31).

4.3. Proof of Theorem 9. Since we have proved Proposition 11, Theorem 9 will follow from the approach of [NS97] and [BS11], as we sketch below. First notice that the Hessian matrix $D^{2} \tilde{H} \geq c_{-} I$.

For any $t>0$ and any $\varphi \in \mathcal{C}_{0}^{\infty}(\mathbb{R})$, set $G^{\varepsilon}(t)=\mathbb{E}\left[e^{t\left\langle\tilde{\eta}^{\varepsilon}, \varphi\right\rangle}\right]$. Applying the exponential Brascamp-Lieb inequality (item 2 of Lemma 6), we conclude that for $t \in[0,1],\left\{G^{\varepsilon}(t)\right\}_{\varepsilon>0}$ 
is uniformly bounded. The same computation as in Section 1.3 of [NS97] then yields

$$
\begin{aligned}
\frac{d G^{\varepsilon}(t)}{d t} & =\mathbb{E}\left[\left\langle\tilde{\eta}^{\varepsilon}, \varphi\right\rangle e^{t\left\langle\tilde{\eta}^{\varepsilon}, \varphi\right\rangle}\right] \\
& =t \varepsilon^{d} \mathbb{E}\left[\left\langle\Delta \varphi, \mathcal{L}^{-1} \Delta \varphi\right\rangle e^{t\left\langle\tilde{\eta}^{\varepsilon}, \varphi\right\rangle}\right] .
\end{aligned}
$$

By Proposition 14 below, we can write

$$
\frac{d G^{\varepsilon}(t)}{d t}=t\langle\nabla \varphi, \nabla \varphi\rangle G_{\varepsilon}(t)+o_{\varepsilon}(1) t
$$

Integrating with respect to $t$ and letting $\varepsilon$ go to zero completes the proof.

To prove Proposition 14, we need the following $L^{1}$-bound for the spatial derivatives of heat kernel, established in [DD05].

Lemma 13. Let $\eta$ be a stationary elliptic random environment on $\left(\mathbb{Z}^{d}\right)^{*}$. For $v \in \mathbb{Z}^{d}$, let $X^{v}$. be a random walk on $\mathbb{Z}^{d}$ starting at $v$ and with environment $\eta$. We simply write $X^{0}$. as $X$.. Then there exists $C_{1}, c_{2}<\infty$ such that for all $t>1$, the discrete spatial derivative $\nabla_{i} \nabla_{j}$ of the heat kernel $p_{0}^{\eta}(t, x) \doteq \mathbb{P}\left(X_{t}=x\right)$ can be bounded by

$$
\mathbb{E}\left|\nabla_{i} \nabla_{j} p_{0}^{\eta}(t, x)\right| \leq \frac{C_{1}}{t^{1+d / 2}} e^{-\|x\|^{2} / 2 c_{2} t}
$$

for $i, j=1, \ldots, d$. Here

$$
\nabla_{i} \nabla_{j} p_{0}^{\eta}(t, x) \doteq \mathbb{P}\left(X_{t}^{e_{i}}=x+e_{j}\right)-\mathbb{P}\left(X_{t}^{e_{i}}=x\right)-\mathbb{P}\left(X_{t}=x+e_{j}\right)+\mathbb{P}\left(X_{t}=x\right) .
$$

Proposition 14. For all $\varphi \in \mathcal{C}_{0}^{\infty}\left(\mathbb{R}^{2}\right)$, we have

$$
\varepsilon^{d}\left\langle\Delta \varphi, \mathcal{L}^{-1} \Delta \varphi\right\rangle \rightarrow\langle\nabla \varphi, \nabla \varphi\rangle \quad \text { in } L^{2} \text { as } \varepsilon \rightarrow 0
$$

The proof of Proposition 14 follows from the same argument as in Section 4.2 of [BS11], and we omit it here. Roughly speaking, both sides can be written as a time integral over $[0, \infty)$. Then one can choose a large $T$, such that the contribution from $[T, \infty)$ is negligible by the $t^{-(1+d / 2)}$ decay of the spatial derivative of the heat kernel (Lemma 13). On $[0, T]$, the desired convergence follows from Proposition 11 .

Remark 15. If the temperature $\beta^{-1}$ tends to zero at a much faster rate as $\varepsilon \rightarrow 0$, i.e. $\beta(\varepsilon) \gg \varepsilon^{-2 d}$, then one can prove a version of Theorem 2 with periodic boundary conditions, i.e., on a d-dimensional torus $\mathbb{T}_{\varepsilon}^{d}$. This can be done by proving a torus version of Theorem 9, and constructing a coupling similar to the one in Section 3.2. Adapting Theorem 9 to a torus is straightforward: the dynamic environment is still translation invariant and stationary, so the homogenization argument still applies. However, the coupling needs some modification, because on a discrete torus, there exists macroscopic cycles with nonzero winding number. To ensure $\eta=\nabla \Phi$, for some function $\Phi: \mathbb{T}_{\varepsilon}^{d} \rightarrow$ $\mathbb{R}, \eta$ cannot have vortices (defined in (14)) along these macroscopic cycles. When the temperature is low enough, i.e. $\beta(\varepsilon) \gg \varepsilon^{-2 d}$, then Theorems 5 and 1 imply that with high probability, $|\eta(b)| \ll \varepsilon$ for all edges $b \in\left(\mathbb{T}_{\varepsilon}^{d}\right)^{*}$. That rules out the possibility of vortices along macroscopic cycles. Thus one can couple the XY model and gradient Gibbs measures in $\mathbb{T}_{\varepsilon}^{d}$ with high probability.

\section{BOUNDED DOMAINS}

We now establish a version of Theorem 9 for gradient fields in the case of bounded domains with Dirichlet boundary condition. It is also analogous to Theorem 2 which was for XY models in bounded domains. 
Theorem 16. Suppose that $\eta^{\varepsilon, D}$ is the gradient variable associated with the Gibbs measure (7). Let $\tilde{\eta}^{\varepsilon, D}=\sqrt{\beta(\varepsilon)} \eta^{\varepsilon}$, and for all $\varphi \in C_{0}^{\infty}(D)$, define $\left\langle\tilde{\eta}^{\varepsilon, D}, \varphi\right\rangle$ as in (5). Assume (17) and that $\beta(\varepsilon) \rightarrow \infty$ as $\varepsilon \rightarrow 0$. Then

$$
\lim _{\varepsilon \rightarrow 0} \mathbb{E}\left[e^{i t\left\langle\tilde{\eta}^{\varepsilon, D}, \varphi\right\rangle}\right]=\exp \left[-\frac{t^{2}}{2}\langle\nabla \varphi, \nabla \varphi\rangle\right] \text {. }
$$

To study the Gibbs measure (7), one can apply a version of the Helffer-Sjöstrand representation described in Section 4.1, and thus reduce the problem to a random walk in a dynamical random environment, that is killed on the boundary. However, as opposed to the infinite volume case, the random environment is no longer translation invariant; thus the homogenization argument used to prove Proposition 11 no longer applies. This difficulty is resolved in [Mil11] by constructing an approximate harmonic coupling for the Gibbs measure, as we discuss next. The approximate harmonic coupling indicates that the Gibbs measure is "Gaussian-like", because it holds exactly for the discrete Gaussian free field (DGFF): the law of a DGFF on some domain $D$ with boundary condition $f$ is equal in law to that of a zero boundary DGFF on $D$, plus the discrete harmonic extension of $f$ to $D$.

We now state the approximate harmonic coupling established in Mil11. Let $D \subset$ $\varepsilon \mathbb{Z}^{2}$ be a bounded subset that approximates a smooth simply connected domain. For $x, y \in D$, let $\operatorname{dist}(x, y)$ be the graph distance between $x$ and $y$, and we denote $D(r)=$ $\{x \in D: \operatorname{dist}(x, \partial D)>r\}$. The Ginzburg-Landau measure on $D$ with Dirichlet boundary condition $f$ is defined by

$$
d \nu^{f}=Z^{-1} \exp \left[-\sum_{(x, y) \in D^{*}} V(\theta(y)-\theta(x))\right] \prod_{x \in D \backslash \partial D} d \theta(x) \prod_{x \in \partial D} \delta(\theta(x)-f(x)) .
$$

Theorem 17 ([Mil1]). Suppose there exists $\Lambda>0$, such that $f: \partial D \rightarrow \mathbb{R}$ satisfies $\max _{x \in \partial D}|f(x)| \leq \Lambda|\log \varepsilon|^{\Lambda}$. Let $\theta$ be sampled from the Ginzburg-Landau measure (37) on $D$ with zero boundary condition, and $\theta^{f}$ be sampled from Ginzburg-Landau measure on $D$ with boundary condition $f$. Assume $V$ satisfies (17) for some $0<c_{-}<c_{+}<\infty$. Then there exist constants $c, \gamma, \delta>0$, that only depends on $c_{-}, c_{+}$, so that if $r>c \varepsilon^{\gamma}$ then the following holds. There exists a coupling $\left(\theta, \theta^{f}\right)$, such that if $\hat{h}: D(r) \rightarrow \mathbb{R}$ is discrete harmonic with $\left.\hat{h}\right|_{\partial D(r)}=\theta^{f}-\left.\theta\right|_{\partial D(r)}$, then

$$
\mathbb{P}\left(\theta^{f}-\theta \neq \hat{h} \text { in } D(r)\right) \leq c(\Lambda) \varepsilon^{\delta} .
$$

Corollary 18. Suppose $V=V_{\varepsilon}$ is such that $\inf _{\varepsilon>0} c_{-}^{\varepsilon}>0$ and $\sup _{\varepsilon>0} c_{+}^{\varepsilon}<\infty$, then the same conclusions are valid with $c, \gamma, \delta$ chosen independent of $\varepsilon$.

Proof. The only difference between Theorem 17 and our current setting is that now $\tilde{V}$ may depend on $\varepsilon$, in a way that

$$
0<c_{-} \leq \inf _{\varepsilon>0} \inf _{x \in \mathbb{R}} \tilde{V}_{\varepsilon}^{\prime \prime}(x) \leq \sup _{\varepsilon>0} \sup _{x \in \mathbb{R}} \tilde{V}_{\varepsilon}^{\prime \prime}(x) \leq c_{+}<\infty .
$$

However, by keeping track of the proof in [Mil11, when $\theta$ and $\theta^{f}$ are sampled from the Gibbs measure (17) with nearest neighbor potential $\tilde{V}_{\varepsilon}$ that satisfies (138), we claim that one can choose corresponding constants $(c, \gamma, \delta)$ that only depend on $c_{-}$and $c_{+}$(and not on $\varepsilon$ ). This claim is implicit in [Mil11]; we sketch here the reasoning. Roughly speaking, denote by $Q$ the law of $\theta+\hat{h}$, the argument in [Mil11] is by controlling the symmetrized relative entropy between $Q$ and $\nu^{f}$, showing that it can be bounded by $c(\Lambda) \varepsilon^{\delta}$. The symmetrized 
relative entropy can be written as a summation over all edges $b \in\left(D^{\varepsilon}\right)^{*}$. For the edges in the bulk $\left(b \in(D(r))^{*}\right)$, one may introduce a coupling between two random walks on random conductances, arising from the Helffer-Sjöstrand representation of the Gibbs measure with different boundary conditions. The bulk term contributes an error $c(\Lambda) \varepsilon^{\delta_{1}}$, where $\delta_{1}$ only depends on the Nash continuity constant of the RWRC (thus only depends on $c_{-}, c_{+}$). For the remaining boundary terms, one can gain some regularity of the field $\theta^{f}-\theta$ by moving a mesoscopic distance $\left(c \varepsilon^{\gamma}\right)$ away from the boundary, and using an energy inequality to bound the error by $c(\Lambda) \varepsilon^{\delta_{2}}$, where $\gamma$ and $\delta_{2}$ only depend on constants in the Beurling type estimates for RWRC (thus only depend on $c_{-}, c_{+}$). Therefore, one can take $\delta=\max \left\{\delta_{1}, \delta_{2}\right\}$, that only depends on $c_{-}$and $c_{+}$. This completes our sketch.

For the rest of this section, we will slightly change the notation, and use superscripts to distinguish the quantities associated with finite volume fields. Let $\left\{\theta^{\varepsilon, D}\right\}$ be sampled from the finite volume Gibbs measure (17), and $\left\{\eta^{\varepsilon}\right\}$ be sampled from the infinite volume gradient Gibbs measure (16). Fix an $x_{0} \in \partial D$, and let $x^{*}$ be the vertex in $D^{\varepsilon}$ with minimal distance to $x_{0}$. We construct a Gibbs measure on $\varepsilon \mathbb{Z}^{d}$ pinned at $x^{*}$, denoted as $\theta_{0}^{\varepsilon}$, by setting $\theta_{0}^{\varepsilon}\left(x^{*}\right)=0$. For any $x \in \varepsilon \mathbb{Z}^{2}$, take a chain $\mathcal{C}_{x^{*}, x}$ connecting $x^{*}$ to $x$, and let $\theta_{0}^{\varepsilon}(x)=\sum_{b \in \mathcal{C}_{x^{*}, x}} \eta^{\varepsilon}(b)$. Define $\tilde{\theta}_{0}^{\varepsilon}=\sqrt{\beta(\varepsilon)} \theta_{0}^{\varepsilon}, \tilde{\theta}^{\varepsilon, D}=\sqrt{\beta(\varepsilon)} \theta^{\varepsilon, D}$. We would like to apply Theorem 17 to couple $\left(\tilde{\theta}_{0}^{\varepsilon}, \tilde{\theta}^{\varepsilon, D}\right)$ and thus also couple $\left(\tilde{\eta}^{\varepsilon}, \tilde{\eta}^{\varepsilon, D}\right)$. To do this, we need to check that the a-priori bound for $\tilde{\theta}_{0}^{\varepsilon}$ on $\partial D^{\varepsilon}$ holds with high probability.

Lemma 19. There exists some $\Lambda<\infty$ such that

$$
\mathbb{P}\left(\max _{x \in \partial D^{\varepsilon}}\left|\tilde{\theta}_{0}^{\varepsilon}(x)\right| \leq \Lambda|\log \varepsilon|^{\Lambda}\right)=1-O\left(\varepsilon^{8}\right) .
$$

Proof. This follows from the same argument as Lemma 7.4 of [Mil11. That is, since $\inf _{\varepsilon>0} \inf _{x} \tilde{V}^{\prime \prime}(x)>c_{-}>0$, we can apply the exponential Brascamp-Lieb inequality (Lemma 6) to control the tail probability of each $\tilde{\theta}_{0}^{\varepsilon}(x)$, and take a union bound. See [Mil11 for more details.

We are now in a position to finish the proof of Theorem 16, following the approach in Mil11.

Proof of Theorem 16. Recall that Theorem 9 implies that for all $\varphi \in C_{0}^{\infty}(D), t \in \mathbb{R}$,

$$
\lim _{\varepsilon \rightarrow 0} \mathbb{E}\left[e^{i t\left\langle\tilde{\eta}^{\varepsilon}, \varphi\right\rangle}\right]=\exp \left[-\frac{t^{2}}{2}\langle\nabla \varphi, \nabla \varphi\rangle\right] .
$$

By Corollary 18 and Lemma 19, on an event $\mathcal{H}_{\varepsilon}$ with probability $1-O\left(\varepsilon^{8}\right)-O\left(\varepsilon^{\delta}\right)$, we can apply Corollary 18 to construct a coupling of $\left(\tilde{\theta}^{\varepsilon}, \tilde{\theta}^{\varepsilon, D}\right)$. More precisely, there exist $\gamma, \delta>0$, independent of $\varepsilon$, such that if we let $\hat{h}^{\varepsilon}$ be the harmonic extension of $\tilde{\theta}_{0}^{\varepsilon}-\tilde{\theta}^{\varepsilon, D}$ from $\partial\left(D^{\varepsilon}\left(\varepsilon^{\gamma}\right)\right)$ to $D^{\varepsilon}\left(\varepsilon^{\gamma}\right)$, on $\mathcal{H}_{\varepsilon}^{c}$ we have $\tilde{\theta}^{\varepsilon}-\tilde{\theta}^{\varepsilon, D}=\hat{h}^{\varepsilon}$ in $D^{\varepsilon}\left(\varepsilon^{\gamma}\right)$, and $\mathbb{P}\left(\mathcal{H}_{\varepsilon}^{c}\right)=O\left(\varepsilon^{\delta}+\varepsilon^{8}\right)$. $\mathcal{H}_{\varepsilon}$,

Given $\varphi \in C_{0}^{\infty}(D)$, we can choose $\varepsilon$ small enough so that $\operatorname{supp}(\varphi) \subset D^{\varepsilon}\left(\varepsilon^{\gamma}\right)$. Then on

$$
\begin{aligned}
\left\langle\tilde{\eta}^{\varepsilon, D}, \varphi\right\rangle & =\left\langle\tilde{\eta}^{\varepsilon}, \varphi\right\rangle-\varepsilon^{d / 2-1} \sum_{b \in\left(D^{\varepsilon}\right)^{*}} \nabla \varphi(b) \nabla\left(\tilde{\theta}_{0}^{\varepsilon}-\tilde{\theta}^{\varepsilon, D}\right) \\
& =\left\langle\tilde{\eta}^{\varepsilon}, \varphi\right\rangle-\varepsilon^{d / 2-1} \sum_{b \in\left(D^{\varepsilon}\right)^{*}} \nabla \varphi(b) \nabla \hat{h}^{\varepsilon}(b) \\
& =\left\langle\tilde{\eta}^{\varepsilon}, \varphi\right\rangle
\end{aligned}
$$


where to obtain the last equality, we apply summation by parts and use the harmonicity of $\hat{h}^{\varepsilon}$. Since $\max \left\{\left|\mathbb{E}\left[e^{i t\left\langle\tilde{\eta}^{\varepsilon}, \varphi\right\rangle} 1_{\mathcal{H}_{\varepsilon}^{c}}\right]\right|,\left|\mathbb{E}\left[e^{i t\left\langle\tilde{\eta}^{\varepsilon, D}, \varphi\right\rangle} 1_{\mathcal{H}_{\varepsilon}^{c}}\right]\right|\right\} \leq \mathbb{P}\left(\mathcal{H}_{\varepsilon}^{c}\right)$, which goes to 0 as $\varepsilon \rightarrow 0$, this implies

$$
\begin{aligned}
\lim _{\varepsilon \rightarrow 0} \mathbb{E}\left[e^{i t\left\langle\tilde{\eta}^{\varepsilon, D}, \varphi\right\rangle}\right] & =\lim _{\varepsilon \rightarrow 0} \mathbb{E}\left[e^{i t\left\langle\tilde{\eta}^{\varepsilon, D}, \varphi\right\rangle} 1_{\mathcal{H}_{\varepsilon}}\right]=\lim _{\varepsilon \rightarrow 0} \mathbb{E}\left[e^{i t\left\langle\tilde{\eta}^{\varepsilon}, \varphi\right\rangle} 1_{\mathcal{H}_{\varepsilon}}\right] \\
& =\lim _{\varepsilon \rightarrow 0} \mathbb{E}\left[e^{i t\left\langle\tilde{\eta}^{\varepsilon}, \varphi\right\rangle}\right] \\
& =\exp \left[-\frac{t^{2}}{2}\langle\nabla \varphi, \nabla \varphi\rangle\right] .
\end{aligned}
$$

Proof of Theorem 2. By an abuse of notation, we denote by $\tilde{\eta}_{X Y}^{\varepsilon, D}$ the rescaled gradient variable associated with the XY model in $D^{\varepsilon}$. Recall the coupling between the XY Gibbs measure (11) and the gradient Gibbs measure (7), established in Section 3.2. We have

$$
\left|\mathbb{E}\left[e^{i t\left\langle\tilde{\eta}_{X Y}^{\varepsilon, D}, \varphi\right\rangle}\right]-\mathbb{E}\left[e^{i t\left\langle\tilde{\eta}^{\varepsilon, D}, \varphi\right\rangle}\right]\right| \leq 2 \mathbb{P}\left(\tilde{\eta}_{X Y}^{\varepsilon, D} \neq \tilde{\eta}^{\varepsilon, D}\right) \leq 2 \mu(\mathcal{B}) .
$$

Therefore, Theorem 2 follows from Theorem 16, and the fact that $\mu(\mathcal{B}) \rightarrow 0$ as $\varepsilon \rightarrow 0$.

\section{Open Questions and Future Work}

6.1. Relaxing the conditions on $\beta(\varepsilon)$. Theorem 2 is in the regime where the temperature $\beta^{-1}$ goes to zero rapidly as the lattice spacing goes to zero. Note that by Remark 8. $\beta(\varepsilon) \gg-9 d \log \varepsilon$ ensures that the XY model is "vortex free" in a domain with diameter $1 / \varepsilon$. When $\beta(\varepsilon) \rightarrow \infty$ at a slower rate, there will still only be a small fraction of plaquettes that form vortices. However, as was discussed in [KT73] and [FS81], at low temperature, positive vortices (i.e., plaquettes $P$ such that $k_{P}>0$ in (3) ) and negative vortices tend to bind to each other, like in the dipole gas. This indicates that at low temperature, macroscopic loops are unlikely to be vortices for the XY model. We therefore expect the conclusion of Theorem 2 to hold as long as $\beta(\varepsilon) \rightarrow \infty$ when $\varepsilon \rightarrow 0$.

6.2. XY model in an external field. Given $h>0$, consider the XY model in $D^{\varepsilon}$ with magnetic field $h$, given by

$$
\begin{aligned}
d \mu_{\beta, h}^{\varepsilon}= & Z_{\beta, h}^{-1} \exp \left[\beta \sum_{(i, j) \in\left(D^{\varepsilon}\right)^{*}} \cos \left(\theta^{\varepsilon}(i)-\theta^{\varepsilon}(j)\right)+h \sum_{i \in D^{\varepsilon}} \cos \theta^{\varepsilon}(i)\right] \\
& \times \prod_{i \in D^{\varepsilon} \backslash \partial D^{\varepsilon}} d \theta^{\varepsilon}(i) \prod_{i \in \partial D^{\varepsilon}} \delta_{0}\left(d \theta^{\varepsilon}(i)\right) .
\end{aligned}
$$

The Gibbs measure (39) fits into the general framework studied by [PL74], and their Theorem 2 implies that the spin-spin correlation decays exponentially with rate at least proportional to $h$. However, the spin wave picture (see e.g. [MW66] and [FS81]) suggests that adding an external field $h$ is like adding a mass proportional to $\sqrt{h}$ to the GFF, and the actual decay rate should be proportional to $\sqrt{h}$ for small $h$. Progress towards this question in $d=3$ has recently been announced [BFS]. Another step would be to understand the zero temperature limit, e.g., by proving the following.

Conjecture 20. Suppose that $\eta^{\varepsilon}$ is the gradient field (as defined in (2)) associated with the Gibbs measure (39), and suppose $\beta=\beta(\varepsilon)$ satisfies (4). Then for any $t \in \mathbb{R}$ and 
$\varphi \in C_{0}^{\infty}(D)$

$$
\lim _{\varepsilon \rightarrow 0} \mathbb{E}\left[e^{i t\left\langle\tilde{r}^{\varepsilon}, \varphi\right\rangle}\right]=\exp \left[-\frac{t^{2}}{2}\left\langle\Delta \varphi,\left(\Delta_{\sqrt{h}}\right)^{-1} \Delta \varphi\right\rangle\right]
$$

where $\tilde{\eta}^{\varepsilon}=\sqrt{\beta} \eta^{\varepsilon}$, and for $m>0$,

$$
\Delta_{m}=\Delta_{0}+m^{2}
$$

where $\Delta_{0}$ is the Dirichlet Laplacian on D.

When $\beta(\varepsilon) \gg-9 d \log \varepsilon$, one can still couple the Gibbs measure (39) with a massive gradient Gibbs measure. The Helffer-Sjöstrand representation still applies and leads to a random walk in a random conductance with exponential killing times. The main technical difficulty is to establish some kind of massive harmonic coupling (analogous to Theorem 17) for massive gradient fields.

Acknowledgments: We thank Thomas Spencer for useful discussions, S.R.Srinivasa Varadhan for discussion of [KV86], Aernout van Enter for useful communications and comments on an earlier version of the paper and Xin Sun for useful communications. The research of C.M.N. and W.W. was supported in part by U.S. NSF grants DMS-1007524 and DMS-1507019.

\section{REFERENCES}

[BF81] Jean Bricmont and Jean-Raymond Fontaine. Correlation inequalities and contour estimates. Journal of Statistical Physics, 26(4):745-753, 1981.

$\left[\mathrm{BFL}^{+} 80\right]$ Jean Bricmont, Jean-Raymond Fontaine, Joel L Lebowitz, Elliott H Lieb, and Thomas Spencer. Lattice systems with a continuous symmetry. iii. low temperature asymptotic expansion for the plane rotator model. Communications in Mathematical Physics, 78(4):545-566, 1980.

[BFS] David Brydges, Jürg Fröhlich, and Thomas Spencer. In preparation.

[Bis09] Marek Biskup. Reflection positivity and phase transitions in lattice spin models. In Methods of Contemporary Mathematical Statistical Physics, pages 1-86. Springer, 2009.

[BL76] Herm Jan Brascamp and Elliott H Lieb. On extensions of the Brunn-Minkowski and PrékopaLeindler theorems, including inequalities for log concave functions, and with an application to the diffusion equation. Journal of Functional Analysis, 22(4):366-389, 1976.

[BS11] Marek Biskup and Herbert Spohn. Scaling limit for a class of gradient fields with nonconvex potentials. The Annals of Probability, 39(1):224-251, 2011.

[DD05] T Delmotte and J-D Deuschel. On estimating the derivatives of symmetric diffusions in stationary random environment, with applications to $\nabla \phi$ interface model. Probability Theory and Related Fields, 133(3):358-390, 2005.

[DGI00] Jean-Dominique Deuschel, Giambattista Giacomin, and Dmitry Ioffe. Large deviations and concentration properties for $\nabla \phi$ interface models. Probability Theory and Related Fields, 117(1):49-111, 2000.

[Dys56] Freeman J Dyson. General theory of spin-wave interactions. Physical Review, 102(5):1217, 1956.

[FS81] Jürg Fröhlich and Thomas Spencer. The Kosterlitz-Thouless transition in two-dimensional abelian spin systems and the Coulomb gas. Communications in Mathematical Physics, 81(4):527-602, 1981.

[FS97] T Funaki and Herbert Spohn. Motion by mean curvature from the Ginzburg-Landau interface model. Communications in Mathematical Physics, 185(1):1-36, 1997.

[FSS76] Jürg Fröhlich, Barry Simon, and Thomas Spencer. Infrared bounds, phase transitions and continuous symmetry breaking. Communications in Mathematical Physics, 50(1):79-95, 1976.

[GK80] K Gawedzki and A Kupiainen. A rigorous block spin approach to massless lattice theories. Communications in Mathematical Physics, 77(1):31-64, 1980.

[GOS01] Giambattista Giacomin, Stefano Olla, and Herbert Spohn. Equilibrium fluctuations for $\nabla \phi$ interface model. Annals of Probability, pages 1138-1172, 2001. 
[Hel82] Inge S Helland. Central limit theorems for martingales with discrete or continuous time. Scandinavian Journal of Statistics, pages 79-94, 1982.

[HS94] Bernard Helffer and Johannes Sjöstrand. On the correlation for Kac-like models in the convex case. Journal of Statistical Physics, 74(1-2):349-409, 1994.

[KT73] John Michael Kosterlitz and David James Thouless. Ordering, metastability and phase transitions in two-dimensional systems. Journal of Physics C: Solid State Physics, 6(7):1181, 1973.

[KV86] Claude Kipnis and SR Srinivasa Varadhan. Central limit theorem for additive functionals of reversible markov processes and applications to simple exclusions. Communications in Mathematical Physics, 104(1):1-19, 1986.

[Mil1] Jason Miller. Fluctuations for the Ginzburg-Landau $\nabla \phi$ interface model on a bounded domain. Communications in Mathematical Physics, 308(3):591-639, 2011.

[MS77] Oliver A McBryan and Thomas Spencer. On the decay of correlations in SO (n)-symmetric ferromagnets. Communications in Mathematical Physics, 53(3):299-302, 1977.

[MW66] N David Mermin and Herbert Wagner. Absence of ferromagnetism or antiferromagnetism in one-or two-dimensional isotropic Heisenberg models. Physical Review Letters, 17(22):1133, 1966.

[NS97] Ali Naddaf and Thomas Spencer. On homogenization and scaling limit of some gradient perturbations of a massless free field. Communications in Mathematical Physics, 183(1):55-84, 1997.

[PL74] O Penrose and JL Lebowitz. On the exponential decay of correlation functions. Communications in Mathematical Physics, 39(3):165-184, 1974.

[Shl86] Semen Bensionovich Shlosman. The method of reflection positivity in the mathematical theory of first-order phase transitions. Russian Mathematical Surveys, 41(3):83-134, 1986.

(Charles Newman) Courant Institute of Mathematical Sciences, New York University, 251 Mercer st, New York, NY 10012, USA, \& NYU-ECNU Institute of Mathematical Sciences at NYU Shanghai, 3663 Zhongshan Road North, Shanghai 200062, China.

(Wei Wu) Courant Institute of Mathematical Sciences, New York University, 251 MerCer st, New York, NY 10012, USA, \& NYU-ECNU Institute of Mathematical Sciences at NYU Shanghai, 3663 Zhongshan Road North, Shanghai 200062, China. 